\title{
Optimal Systems and Invariant Solutions for the Curve Shortening Problem
}

\author{
KaI-Seng Chou and Guan-Xin Li
}

The symmetry group of the generalized curve shortening problem is determined and a corresponding optimal system is found. Group invariant solutions for the optimal system are discussed.

\section{Introduction.}

In the past fifteen years there has been constant progress in the study of the curve shortening problem. From a geometric point of view, this is the simplest evolution equation by which people employ to deform geometric objects to canonical ones. On the other hand, it also appears to be useful in describing the motion of the sharp interface in phase transition. Analytically it is a weakly parabolic system for a curve $\gamma(\cdot, t)$

$$
\frac{\partial \gamma}{\partial t}=k n
$$

where $\boldsymbol{n}$ and $k$ are respectively a choice of unit normal for $\gamma$ and the curvature with respect to $\boldsymbol{n}$. For a simple closed curve it was shown in GageHamilton [15] and Grayson [16] that the curve will evolve into a convex one and then shrink to a point asymptotic to a circle. So the problem is wellunderstood in this case. However, for a closed immersed curve it is known that singularities may develop before it shrinks to a point. The analysis of the singularities is difficult and is not completed. Some progress has been made, for example, in Altschuler [2], Angenent [6], [7], [8], Huisken [18] and Oaks [21]. It turns out that certain group invariant solutions are crucial for the study. For instance, it was shown that when a locally convex closed immersed curve collapses into a point, its asymptotic shape must be one of the contracting self-similar solutions of (1) classified in Abresch-Langer [1]. On the other hand, the asymptotic profile for a "type-II singularity" looks like a "grim reaper", a traveling wave solution first observed in [15]. In fact, the "grim reaper" was also used by Hamilton [17] to give an alternate proof of the main result in [14] and [15]. Finally, a contracting spiral solution was 
used in [8] in the analysis of type-I and type-II singularities.

Problem (1) also makes sense for complete, noncompact curves. In [14] Ecker and Huisken established a long time existence result when the initial curve is a graph over the real line. (Actually, they also proved the result for the evolution of entire graphs for the mean curvature flow.) For a class of graphs of slow growth they showed that they are asymptotic to expanding self-similar solutions of (1). In a recent work, the first author and Zhu [12] have proved a long time existence result for a rather general class of complete, noncompact curves. It is the question of long time behavior of these curves that leads us to the present work.

In this work we attempt to give a systematic investigation on the group invariant solutions of the generalized curve shortening problem

$$
\frac{\partial \gamma}{\partial t}=|k|^{\sigma-1} k n, \quad \sigma>0 .
$$

When $\sigma=1 / 3$, the problem is called affine curve shortening and is related to image processing (Alvarez, Guichard, Lions and Morel [3], Andrews [5], Angenent-Sapiro-Tannenbaum [9] and Sapiro-Tannenbaum [25], [26]). The problem for general $\sigma$ has been studied by Andrews [4] recently. To proceed our discussion further it is necessary to fix a parametrization of (2). Let's assume $\gamma=(x, u(x, t))$. Then (2) becomes the non-uniformly parabolic equation

$$
u_{t}=\left|\frac{u_{x x}}{\left(1+u_{x}^{2}\right)^{3 / 2}}\right|^{\sigma-1} \frac{u_{x x}}{1+u_{x}^{2}}, \sigma>0 .
$$

In Lie's theory of symmetry groups for differential equations a one-parameter group of symmetries is a family of local diffeomorphisms

$$
\begin{aligned}
& \tilde{x}=\Sigma_{\varepsilon}(x, u), \\
& \tilde{u}=\Phi_{\varepsilon}(x, u), \quad \varepsilon \text { small },
\end{aligned}
$$

satisfying $x=\Sigma_{0}(x, u)$ and $u=\Phi_{0}(x, u)$ which preserve solutions of (3). The vector field

where

$$
\boldsymbol{v}=\xi \frac{\partial}{\partial x}+\phi \frac{\partial}{\partial u},
$$

$$
\begin{aligned}
& \xi(x, u)=\left.\frac{\partial}{\partial \varepsilon}\right|_{\varepsilon=0} \Sigma_{\varepsilon}(x, u), \quad \text { and } \\
& \phi(x, u)=\left.\frac{\partial}{\partial \varepsilon}\right|_{\varepsilon=0} \Phi_{\varepsilon}(x, u),
\end{aligned}
$$


is the infinitesimal symmetry for the 1-parameter group. In order to obtain all group invariant solutions of (3) we first determine the Lie algebra of all infinitesimal symmetries. Since the equation is geometric, it must admit the Euclidean motions (translations in $x$ and $u$, rotation in $x-u$ but not the reflection because it is discrete) as its symmetries. Furthermore, being independent of $t$ explicitly means that it admits translation in $t$. Finally, the special form of (3) suggests that it also admits a certain scaling invariance. We shall show that in fact all these infinitesimal symmetries form a basis of all symmetries as long as $\sigma \neq 1 / 3$. When $\sigma=1 / 3$, the Lie algebra of infinitesimal symmetries has seven dimension, two more than the non-affine case. After determining the Lie algebra, we consider the group invariant solutions of (3) and (2). According to the general theory, given any infinitesimal symmetry $\boldsymbol{v}$, or more precisely the subspace spanned by $\boldsymbol{v}$, usually there corresponds a $\boldsymbol{v}$-invariant solution. In order to describe all group invariant solutions, one needs the concept of an optimal system. See Section 1 for details. We shall determine an optimal system for (3). In a certain sense all group invariant solutions are determined. This paper is organized as follows. We carry out the group analysis for (3) and determine an optimal system in the non-affine case in Section 1. In Section 2 we find an optimal system for the affine case. In Section 3 we discuss an affine invariant solutions in some details.

Acknowledgement. The support of an Earmarked Grant for Research, H.K., is acknowledged. We would like to thank Dr. K.S. Li for preparing the figures in this paper.

\section{An Optimal System for $\sigma \neq 1 / 3$.}

Consider the generalized curve shortening problem (2). In this section we determine an optimal system for non-affine (2). First of all, we observe that this system is geometric; any reparametrization of the curve leaves it invariant. As a result, any diffeomorphism on the parameter space is a symmetry for (2). To get rid of these reduntant symmetries we must fix a parametrization. From the analytic point of view, this is also useful since it reduces the weakly parabolic system (2) to a single parabolic equation. A frequently used parametrization is to represent $\gamma$ as graphs. Let's assume that during some time interval, $\gamma(\cdot, t)$ is the graph $(x, u(x, t)), x \in(a, b)$. We may choose the orientation of $\gamma$ so that it is along the positive $x$-axis. Then 
the unit normal and curvature of $\gamma(\cdot, t)$ are given by the formulas

$$
\boldsymbol{n}=\frac{1}{\sqrt{1+u_{x}^{2}}}\left(-u_{x}, 1\right)
$$

and

$$
k=\frac{u_{x x}}{\left(1+u_{x}^{2}\right)^{3 / 2}},
$$

respectively. The normal velocity of $\gamma$ is given by

$$
\left(0, u_{t}\right) \cdot \boldsymbol{n}=u_{t}\left(1+u_{x}^{2}\right)^{-\frac{1}{2}} .
$$

Therefore, (2) becomes (3). Actually, it can be shown that this equation is equivalent to (2). Since our consideration is local in nature, it is without loss of generality to assume $u_{x x}$ is positive. Then it becomes

$$
\begin{aligned}
\Delta[u] & \equiv u_{t}\left(1+u_{x}^{2}\right)^{\frac{3 \sigma-1}{2}}-u_{x x}^{\sigma}, \sigma>0, \\
& =0 .
\end{aligned}
$$

The symmetry group of (1.1) can be obtained by a routine computation using Lie's infinitesimal criterion for symmetry [22].

Proposition 1.1. Denote the vector space of all infinitesimal symmetries of $(1.1)$ by $\mathfrak{s}(\sigma)$. Then

(a) when $\sigma \neq 1 / 3, \mathfrak{s}(\sigma)$ is spanned by

$$
\left\{\partial_{x}, \partial_{u},-u \partial_{x}+x \partial_{u}, \partial_{t}, x \partial_{x}+u \partial_{u}+(1+\sigma) t \partial_{t}\right\}
$$

(b) when $\sigma=1 / 3, \mathfrak{s}(\sigma)$ is spanned by

$$
\left\{\partial_{x}, \partial_{u},-u \partial_{x}+x \partial_{u}, u \partial_{x}+x \partial_{u}, x \partial_{x}-u \partial_{u}, \partial_{t}, x \partial_{x}+u \partial_{u}+\frac{4}{3} t \partial_{t}\right\}
$$

From now on we shall denote

$$
\begin{array}{ll}
\boldsymbol{v}_{1}=\partial_{x} & (\text { translation in } x) \\
\boldsymbol{v}_{2}=\partial_{u} & (\text { translation in } u) \\
\boldsymbol{v}_{3}=-u \partial_{x}+x \partial_{u} & (\text { rotation from } x \text { - to } u \text {-axis }) \\
\boldsymbol{v}_{4}=u \partial_{x}+x \partial_{u} & \\
\boldsymbol{v}_{5}=x \partial_{x}-u \partial_{u} & (\text { translation in } t) \\
\boldsymbol{t}=\partial_{t} & (\text { dilatation })
\end{array}
$$


Table 1: Composition for $\mathfrak{s}(\sigma)$. The $(i, j)$ - entry is $\left[\boldsymbol{v}_{i}, \boldsymbol{v}_{j}\right]$.

\begin{tabular}{|c|c|c|c|c|c|c|c|}
\hline $\mathfrak{s}(\sigma)$ & $\boldsymbol{v}_{1}$ & $\boldsymbol{v}_{2}$ & $\boldsymbol{v}_{3}$ & $\boldsymbol{v}_{4}$ & $\boldsymbol{v}_{5}$ & $\boldsymbol{t}$ & $\boldsymbol{d}$ \\
\hline $\boldsymbol{v}_{1}$ & 0 & 0 & $\boldsymbol{v}_{2}$ & $\boldsymbol{v}_{2}$ & $\boldsymbol{v}_{1}$ & 0 & $\boldsymbol{v}_{1}$ \\
\hline $\boldsymbol{v}_{2}$ & 0 & 0 & $-\boldsymbol{v}_{1}$ & $\boldsymbol{v}_{1}$ & $-\boldsymbol{v}_{2}$ & 0 & $\boldsymbol{v}_{2}$ \\
\hline $\boldsymbol{v}_{3}$ & $-\boldsymbol{v}_{2}$ & $\boldsymbol{v}_{1}$ & 0 & $2 \boldsymbol{v}_{5}$ & $-2 \boldsymbol{v}_{4}$ & 0 & 0 \\
\hline $\boldsymbol{v}_{4}$ & $-\boldsymbol{v}_{2}$ & $-\boldsymbol{v}_{1}$ & $-2 \boldsymbol{v}_{5}$ & 0 & $-2 \boldsymbol{v}_{3}$ & 0 & 0 \\
\hline $\boldsymbol{v}_{5}$ & $-\boldsymbol{v}_{1}$ & $\boldsymbol{v}_{2}$ & $2 \boldsymbol{v}_{4}$ & $2 \boldsymbol{v}_{3}$ & 0 & 0 & 0 \\
\hline $\boldsymbol{t}$ & 0 & 0 & 0 & 0 & 0 & 0 & $(\sigma+1) \boldsymbol{t}$ \\
\hline $\boldsymbol{d}$ & $-\boldsymbol{v}_{1}$ & $-\boldsymbol{v}_{2}$ & 0 & 0 & 0 & $-(\sigma+1) \boldsymbol{t}$ & 0 \\
\hline
\end{tabular}

According to the general theory, $\mathfrak{s}(\sigma)$ forms a Lie algebra under the usual Poisson bracket for vector fields. The structure of $\mathfrak{s}(\sigma)$ is shown in Table 1 .

We shall regard any element of $\mathfrak{s}(\sigma)$ as a vector field in $(x, u, t) \in \mathbb{R}^{3}$. Since it is linear, the one-parameter group of diffeomorphisms it generates is global on $\mathbb{R}^{3}$. Let's denote the Lie group corresponding to $\mathfrak{s}(\sigma)$ by $S(\sigma)$. In fact, it is the connected component of the identity of the full symmetry group of (1.1). Every element $g$ in $S(\sigma)$ is a symmetry of (2) in the sense that $g(\gamma(\cdot, t), t)$ is a solution of $(2)$ whenever $(\gamma(\cdot, t), t)$ is a solution. The structure of $S(\sigma)$ is given by the following proposition.

Proposition 1.2. Every element in $S(\sigma), \sigma \neq 1 / 3$, can be expressed as

$$
g=D T E
$$

where $E$ is a rigid motion on the plane, $T$ is a translation in $t$ and $D$ is a dilatation: $(x, u, t) \mapsto\left(e^{\varepsilon} x, e^{\varepsilon} u, e^{(\sigma+1) \varepsilon} t\right)$ for some $\varepsilon$.

Proof. Observe that $\boldsymbol{v}_{1}, \boldsymbol{v}_{2}$ and $\boldsymbol{v}_{3}$ generate the Euclidean group of rigid motions on the plane and act trivially on the $t$-component. Using the relations

$$
\begin{aligned}
& T D=D T^{\prime}, \quad T^{\prime} \text { a translation in } t \\
& T E=E T
\end{aligned}
$$


it is easy to see that every finite product of $\exp \varepsilon_{i} \boldsymbol{v}_{i}, i=1, \ldots, 3$, exp $\varepsilon_{4} \boldsymbol{t}$, and exp $\varepsilon_{5} d$, can be expressed in the form $D T E$. Since $S(\sigma)$ is a connected Lie group and so each of its elements can be represented by a finite product of this form, the proposition holds.

For each one-dimensional subalgebra $\mathfrak{h}$ of $\mathfrak{s}(\sigma)$, there corresponds a oneparameter subgroup of $S(\sigma)$ given by

$$
H=\{\exp \varepsilon \boldsymbol{v}: \varepsilon \in \mathbb{R}\},
$$

where $\boldsymbol{v}$ is any nonzero element of $\mathfrak{h}$. According to Lie's theory, there is a group invariant solution, called $H$ or $\mathfrak{h}$-invariant, associated to each $\mathfrak{h}$. The group invariant solution satisfies a "reduced equation", which, in our situation, is a second order ordinary differential equation. Let's take $\boldsymbol{v}=$ $c \partial_{u}+\partial_{t}(c \neq 0)$ as an example. By solving the $O D E$

$$
\frac{d x}{0}=\frac{d u}{c}=\frac{d t}{1}=d \varepsilon
$$

we obtain the group action

$$
\begin{aligned}
h_{\varepsilon}(x, u, t) & =\exp \varepsilon \boldsymbol{v}(x, u, t) \\
& =(x, u+c \varepsilon, t+\varepsilon), \varepsilon \in \mathbb{R} .
\end{aligned}
$$

There are two invariants: $x$ and $u-c t$. Setting $u(x, t)=c t+v(x)$ in (1.2), we see that $v(x)$ satisfies the equation

$$
v_{x x}^{\sigma}=c\left(1+v_{x}^{2}\right)^{\frac{3 \sigma-1}{2}} .
$$

By solving this equation we obtain a $\boldsymbol{v}$-invariant solution which is in fact a traveling wave.

Since there are infinitely many one-dimensional subalgebras, it is impossible to write down all reduced equations, let alone solving them. Following Ovsiannikov [23] , we shall classify them. A good discussion on optimal systems can be found in Chapter 3 in [22]. We say two subalgebras $\mathfrak{h}_{1}$ and $\mathfrak{h}_{2}$ are equivalent if there exists $g \in S(\sigma)$ such that $A d g\left(\mathfrak{h}_{1}\right)=\mathfrak{h}_{2}$. Here $A d g$ is the adjoint representation of $g$ on $\mathfrak{s}(\sigma)$. It is a linear isomorphism on $\mathfrak{s}(\sigma)$. An optimal system (of one-dimensional subalgebras) is a collection of one-dimensional subalgebras $\left\{\mathfrak{h}_{\alpha}\right\}_{\alpha \in A}$ satisfying (1) for any $\mathfrak{h}$ there exist $g \in S(\sigma)$ and $\mathfrak{h}_{\alpha}$ such that $\operatorname{Ad} g\left(\mathfrak{h}_{\alpha}\right)=\mathfrak{h}$, and (2) all $\mathfrak{h}_{\alpha}^{\prime} s$ are mutually inequivalent. At the group level, the relevance of this notion is that, $(\gamma, t)$ 
is a $H$-invariant solution if and only if $g(\gamma, t)$ is a $g H g^{-1}$-invariant solution. See, e.g., Proposition 3.6 of [22]. Therefore, once an optimal system is constructed, all group invariant solutions can be obtained as images of the $\mathfrak{h}_{\alpha}$-invariant solution $(\alpha \in A)$ under $A d S(\sigma)$.

Now we determine an optimal system for (1.1) in the non-affine case.

Theorem 1.3. When $\sigma \neq 1 / 3$, an optimal system consists of 1-dimensional subalgebras generated by

$$
\begin{aligned}
& \boldsymbol{w}_{1}=\boldsymbol{v}_{1} \\
& \boldsymbol{w}_{2}=\boldsymbol{t} \\
& \boldsymbol{w}_{3}=\boldsymbol{v}_{2}+\boldsymbol{t} \\
& \boldsymbol{w}_{4}=\boldsymbol{v}_{3} \\
& \boldsymbol{w}_{5}=\boldsymbol{v}_{3}+\boldsymbol{t} \\
& \boldsymbol{w}_{6}=\boldsymbol{v}_{3}-\boldsymbol{t} \\
& \boldsymbol{w}_{7}=\boldsymbol{d} \\
& \boldsymbol{w}_{8}=\boldsymbol{d}+\alpha \boldsymbol{v}_{3}, \alpha \neq 0 .
\end{aligned}
$$

Notice that $\boldsymbol{w}_{8}$ contains infinitely many elements. We remark that when $\sigma=1,(1.1)$ arises in nonlinear filtration and an optimal system for (1.1) is listed without proof in Chapter 10 of the handbook [19].

Proof. The action of the adjoint representation of $S(\sigma)$ on $\mathfrak{s}(\sigma)$ is shown in Table 2, discarding $\boldsymbol{v}_{4}$ and $\boldsymbol{v}_{5}$ for the moment. It is obtained by using the formula

$$
A d(\exp (\varepsilon \boldsymbol{v})) \boldsymbol{w}=\boldsymbol{w}-\varepsilon[\boldsymbol{v}, \boldsymbol{w}]+\frac{\varepsilon^{2}}{2}[\boldsymbol{v},[\boldsymbol{v}, \boldsymbol{w}]]-\cdots
$$

Let

$$
\boldsymbol{v}=\sum_{i=1}^{3} a_{i} \boldsymbol{v}_{i}+a_{4} \boldsymbol{t}+a_{5} \boldsymbol{d}
$$

be in $\mathfrak{s}(\sigma)$. We consider several cases separately.

Case 1. $a_{3}=a_{5}=0$. If further $a_{4}=0$, we can use $A d\left(\exp \varepsilon \boldsymbol{v}_{3}\right)$ for a suitably chosen $\varepsilon$ to eliminate $\boldsymbol{v}_{2}$. Hence $\langle\boldsymbol{v}\rangle$ is equivalent to $\left\langle\boldsymbol{v}_{1}\right\rangle$. If $a_{4} \neq 0$, we use $A d\left(\exp \varepsilon \boldsymbol{v}_{3}\right)$ to eliminate $\boldsymbol{v}_{1}$ and then $A d(\exp \varepsilon \boldsymbol{d})$ to normalize the resulting vector. Then $\langle\boldsymbol{v}\rangle$ is equivalent to the space spanned by

$$
\begin{array}{lll}
\boldsymbol{w}_{2}=\boldsymbol{t} & \text { if } & a_{1}=a_{2}=0 \\
\boldsymbol{w}_{3}=\boldsymbol{v}_{2}+\boldsymbol{t} & \text { if } & a_{1}^{2}+a_{2}^{2} \neq 0 .
\end{array}
$$


Table 2: The $(i, j)$ - entry is $A d\left(\exp \varepsilon \boldsymbol{v}_{i}\right) \boldsymbol{v}_{j}$.

\begin{tabular}{|c|c|c|c|c|c|c|c|}
\hline$A d$ & $\boldsymbol{v}_{1}$ & $\boldsymbol{v}_{2}$ & $\boldsymbol{v}_{3}$ & $\boldsymbol{v}_{4}$ & $\boldsymbol{v}_{5}$ & $\boldsymbol{t}$ & $\boldsymbol{d}$ \\
\hline $\boldsymbol{v}_{1}$ & $\boldsymbol{v}_{1}$ & $\boldsymbol{v}_{2}$ & $\boldsymbol{v}_{3}-\varepsilon \boldsymbol{v}_{2}$ & $\boldsymbol{v}_{4}-\varepsilon \boldsymbol{v}_{2}$ & $\boldsymbol{v}_{5}-\varepsilon \boldsymbol{v}_{1}$ & $\boldsymbol{t}$ & $\boldsymbol{d}-\varepsilon \boldsymbol{v}_{1}$ \\
\hline $\boldsymbol{v}_{2}$ & $\boldsymbol{v}_{1}$ & $\boldsymbol{v}_{2}$ & $\boldsymbol{v}_{3}+\varepsilon \boldsymbol{v}_{1}$ & $\boldsymbol{v}_{4}-\varepsilon \boldsymbol{v}_{1}$ & $\boldsymbol{v}_{5}+\varepsilon \boldsymbol{v}_{2}$ & $\boldsymbol{t}$ & $\boldsymbol{d}-\varepsilon \boldsymbol{v}_{2}$ \\
\hline $\boldsymbol{v}_{3}$ & $\boldsymbol{v}_{1} \cos \varepsilon+$ & $\boldsymbol{v}_{2} \cos \varepsilon-$ & $\boldsymbol{v}_{3}$ & $\boldsymbol{v}_{4} \cos 2 \varepsilon-$ & $\boldsymbol{v}_{5} \cos 2 \varepsilon+$ & $\boldsymbol{t}$ & $\boldsymbol{d}$ \\
& $\boldsymbol{v}_{2} \sin \varepsilon$ & $\boldsymbol{v}_{1} \sin \varepsilon$ & $\boldsymbol{v}_{5} \sin 2 \varepsilon$ & $\boldsymbol{v}_{4} \sin 2 \varepsilon$ & & \\
\hline $\boldsymbol{v}_{4}$ & $\boldsymbol{v}_{1} \cosh \varepsilon+$ & $\boldsymbol{v}_{2} \cosh \varepsilon+$ & $\boldsymbol{v}_{3} \cosh 2 \varepsilon+$ & $\boldsymbol{v}_{4}$ & $\boldsymbol{v}_{5} \cosh 2 \varepsilon+$ & $\boldsymbol{t}$ & $\boldsymbol{d}$ \\
$\boldsymbol{v}_{2} \sinh \varepsilon$ & $\boldsymbol{v}_{1} \sinh \varepsilon$ & $\boldsymbol{v}_{5} \sinh 2 \varepsilon$ & $\boldsymbol{v}_{3} \sinh 2 \varepsilon$ & & $\boldsymbol{t}$ \\
\hline $\boldsymbol{v}_{5}$ & $e^{\varepsilon} \boldsymbol{v}_{1}$ & $e^{-\varepsilon} \boldsymbol{v}_{2}$ & $\boldsymbol{v}_{3} \cosh 2 \varepsilon-$ & $\boldsymbol{v}_{4} \cosh 2 \varepsilon-$ & $\boldsymbol{v}_{5}$ & $\boldsymbol{t}$ & $\boldsymbol{d}$ \\
\hline $\boldsymbol{t}$ & $\boldsymbol{v}_{1}$ & $\boldsymbol{v}_{2}$ & $\boldsymbol{v}_{3}$ & $\boldsymbol{v}_{4}$ & $\boldsymbol{v}_{5}$ & $\boldsymbol{t}$ & $\boldsymbol{d}-\frac{4}{3} \varepsilon \boldsymbol{t}$ \\
\hline $\boldsymbol{d}$ & $e^{\varepsilon} \boldsymbol{v}_{1}$ & $e^{\varepsilon} \boldsymbol{v}_{2}$ & $\boldsymbol{v}_{3}$ & $\boldsymbol{v}_{4}$ & $\boldsymbol{v}_{5}$ & $e^{\frac{4}{3} \varepsilon}$ & $\boldsymbol{d}$ \\
\hline
\end{tabular}

Case 2. $a_{3}^{2}+a_{5}^{2} \neq 0$. If $a_{5}=0$, then $a_{3} \neq 0$. We use $A d\left(\exp \varepsilon \boldsymbol{v}_{1}\right)$ and $A d\left(\exp \varepsilon \boldsymbol{v}_{2}\right)$ to eliminate $\boldsymbol{v}_{1}$ and $\boldsymbol{v}_{2}$, and then use $A d(\exp \varepsilon \boldsymbol{d})$ to normalize the vector. Then $\langle\boldsymbol{v}\rangle$ is equivalent to the space spanned by

$$
\begin{array}{lll}
\boldsymbol{w}_{4}=\boldsymbol{v}_{3} & \text { if } & a_{4}=0, \\
\boldsymbol{w}_{5}=\boldsymbol{v}_{3}+\boldsymbol{t} & \text { if } & a_{3} a_{4}>0, \\
\boldsymbol{w}_{6}=\boldsymbol{v}_{3}-\boldsymbol{t} & \text { if } & a_{3} a_{4}<0 .
\end{array}
$$

If $a_{5} \neq 0$, we use $A d\left(\exp \varepsilon \boldsymbol{v}_{1}\right), A d\left(\exp \varepsilon \boldsymbol{v}_{2}\right)$ and $A d(\exp \varepsilon \boldsymbol{t})$ to eliminate $\boldsymbol{v}_{1}, \boldsymbol{v}_{2}$ and $\boldsymbol{t}$. So $\langle\boldsymbol{v}\rangle$ is equivalent to

$$
\begin{array}{lll}
\boldsymbol{w}_{7}=\boldsymbol{d} & \text { if } & a_{3}=0, \\
\boldsymbol{w}_{8}=\boldsymbol{d}+\alpha \boldsymbol{v}_{3}, \alpha \neq 0 & \text { if } & a_{3} \neq 0 .
\end{array}
$$

We have shown that any one-dimensional subspace of $\mathfrak{s}(\sigma)$ is equivalent to one of the subspaces spanned by $\boldsymbol{w}_{1}, \ldots \boldsymbol{w}_{8}$.

Now we claim that they are inequivalent, and hence form an optimal system. To prove this we define some invariants [22]. A mapping $i: \mathfrak{s}(\sigma) \rightarrow$ 
$\mathbb{R}$ is called an invariant if $i(A d g \boldsymbol{v})=i(\boldsymbol{v})$ for all $g \in S(\sigma)$. Notice that any element in $S(\sigma)$ is of the form

$$
\exp \left(\varepsilon_{1} \boldsymbol{v}_{i_{1}}\right) \cdots \exp \left(\varepsilon_{N} \boldsymbol{v}_{i_{N}}\right) \exp (\varepsilon \boldsymbol{t}) \exp \left(\varepsilon^{\prime} \boldsymbol{d}\right) .
$$

So $i$ is an invariant if and only if $i\left(A d\left(\exp \varepsilon \boldsymbol{v}_{i}\right) \boldsymbol{v}\right)=i(\operatorname{Ad}(\exp \varepsilon \boldsymbol{t}) \boldsymbol{v})=$ $i(\operatorname{Ad}(\exp \varepsilon \boldsymbol{d}) \boldsymbol{v})=i(\boldsymbol{v})$ for all $\varepsilon, \boldsymbol{v}_{i}(i=1, \ldots, 3)$ and $\boldsymbol{v}$.

Fact $1 . a_{3}$ and $a_{5}$ are invariants. This can be easily seen from Table 2.

Fact 2. $b$ is an invariant where

$$
b= \begin{cases}1 & a_{3}^{2}+a_{5}^{2}=0, a_{1}^{2}+a_{2}^{2} \neq 0 \\ 0 & a_{3}^{2}+a_{5}^{2}=0, a_{1}^{2}+a_{2}^{2}=0 \\ 0 & a_{3}^{2}+a_{5}^{2} \neq 0\end{cases}
$$

Since $a_{3}^{2}+a_{5}^{2}$ is an invariant, it suffices to check the invariance of $b$ under $a_{3}^{2}+a_{5}^{2}=0$. However, observe that the adjoint actions of $\exp \varepsilon \boldsymbol{v}_{i}, i=1,2$, and $\exp \varepsilon \boldsymbol{t}$ do not change $\boldsymbol{v}_{1}$ and $\boldsymbol{v}_{2}$. We only need to check the actions of $A d \exp \varepsilon \boldsymbol{v}_{3}$ and $A d \exp \varepsilon \boldsymbol{d}$. In fact, after acted by $A d\left(\exp \varepsilon \boldsymbol{v}_{3}\right)$, the new coefficients of $\boldsymbol{v}_{1}$ and $\boldsymbol{v}_{2}$, say $\tilde{a}_{1}$ and $\tilde{a}_{2}$, satisfy $\tilde{a}_{1}^{2}+\tilde{a}_{1}^{2}=a_{1}^{2}+a_{2}^{2}$, and $b$ is unchanged. On the other hand, under $\operatorname{Ad}(\exp \varepsilon \boldsymbol{d})$, the new coefficients of $\boldsymbol{v}_{1}$ and $\boldsymbol{v}_{2}$, say $\tilde{a}_{1}$ and $\tilde{a}_{2}$, satisfy $\tilde{a}_{1}^{2}+\tilde{a}_{2}^{2}=e^{2 \varepsilon}\left(a_{1}^{2}+a_{2}^{2}\right)$. Hence $b$ is also unchanged. We conclude that $b$ is actually an invariant.

Fact 3. $c$ is an invariant where

$$
c= \begin{cases}\operatorname{sign} a_{4} & a_{5}=0, \\ 0 & a_{5} \neq 0 .\end{cases}
$$

This is obvious from Table 2. Now we put everything in the following table. 
Table 3 .

\begin{tabular}{|c|c|c|c|c|}
\hline & $a_{3}$ & $a_{5}$ & $b$ & $c$ \\
\hline $\boldsymbol{w}_{1}$ & 0 & 0 & 1 & 0 \\
\hline $\boldsymbol{w}_{2}$ & 0 & 0 & 0 & 1 \\
\hline $\boldsymbol{w}_{3}$ & 0 & 0 & 1 & 1 \\
\hline $\boldsymbol{w}_{4}$ & 1 & 0 & 0 & 0 \\
\hline $\boldsymbol{w}_{5}$ & 1 & 0 & 0 & 1 \\
\hline $\boldsymbol{w}_{6}$ & 1 & 0 & 0 & -1 \\
\hline $\boldsymbol{w}_{7}$ & 0 & 1 & 0 & 0 \\
\hline $\boldsymbol{w}_{8}$ & $\alpha$ & 1 & 0 & 0 \\
\hline
\end{tabular}

It is clear from Table 3 that $\boldsymbol{w}_{i}^{\prime} s$ are mutually inequivalent. We have established the optimality of $\left\{\boldsymbol{w}_{1}, \ldots, \boldsymbol{w}_{8}\right\}$.

Now we list the reduced equations of the optimal system in the following table. Both reduced equations for $\boldsymbol{w}_{1}$ and $\boldsymbol{w}_{2}(t$-translational invariant and $x$-translational invariant solutions respectively) yield the same solutions, namely, $u(x)=a x+b, a, b \in \mathbb{R}$. Therefore, all invariant solutions equivalent to $\boldsymbol{w}_{1}$ or $\boldsymbol{w}_{2}$ are straight lines. Rotational invariant solutions are solutions to the reduced equation for $\boldsymbol{w}_{4}$ whose general solution is given by

$$
v(t)=[R-(\sigma+1) t]^{\frac{2}{\sigma+1}}, R>0 .
$$

Since $v=x^{2}+u^{2}$, the solutions is a shrinking circle, collapsing to a point at time $=R /(\sigma+1)$ where $R^{\frac{1}{\sigma+1}}$ is the initial radius. The $w_{4}$-invariant solution is a traveling wave called the grim reaper when $\sigma=1$ [16]. Both $\boldsymbol{w}_{5^{-}}$and $\boldsymbol{w}_{6}$-invarient solutions are spirals called yin-yang curves when $\sigma=1$ [2]. Contracting $(t<0)$ and expanding $(t>0)$ self-similar solutions (AbreshLanger [1], Brakke [10] and [14]) correspond to the vector field $\boldsymbol{w}_{7}$. All these solutions are discussed in Chou-Zhu [13]. Finally, the contracting \expanding spirals corresponding to $\boldsymbol{w}_{8}$ are discussed in [8] and Li [20]. 
Table 4.

\begin{tabular}{|c|c|c|c|}
\hline & group action on $(x, u, t)$ & invariant $(y, v)$ & reduced equation \\
\hline$w_{1}$ & $(x+\varepsilon, u, t)$ & $(t, u)$ & $v_{y}=0$ \\
\hline$w_{2}$ & $(x, u, t+\varepsilon)$ & $(x, u)$ & $v_{y y}=0$ \\
\hline$w_{3}$ & $(x, u+\varepsilon, t+\varepsilon)$ & $(x, u-t)$ & $v_{y y}=\left(1+v_{y}^{2}\right)^{\frac{3 \sigma-1}{2 \sigma}}$ \\
\hline$w_{4}$ & $\begin{array}{c}(x \cos \varepsilon-u \sin \varepsilon \\
u \cos \varepsilon+x \sin \varepsilon, t)\end{array}$ & $\left(t, x^{2}+u^{2}\right)$ & $v_{y}=-2 v^{\frac{1-\sigma}{2}}$ \\
\hline$w_{5}$ & $\begin{array}{c}(x \cos \varepsilon-u \sin \varepsilon \\
u \cos \varepsilon+x \sin \varepsilon, t+\varepsilon)\end{array}$ & $\left(x^{2}+u^{2}, \tan ^{-1} u / x-t\right)$ & $\begin{array}{l}v_{y}=\frac{\lambda}{2 y} \\
2 \lambda_{y}=y^{\frac{1-\sigma}{2 \sigma}}\left(1+\lambda^{2}\right)^{\frac{3 \sigma-1}{2 \sigma}} \\
-y^{-1} \lambda\left(1+\lambda^{2}\right)\end{array}$ \\
\hline$w_{6}$ & $\begin{array}{c}(x \cos \varepsilon-u \sin \varepsilon \\
u \cos \varepsilon+x \sin \varepsilon, t-\varepsilon)\end{array}$ & $\left(x^{2}+u^{2}, \tan ^{-1} u / x+t\right)$ & $\begin{array}{l}v_{y}=\frac{\lambda}{2 y} \\
2 \lambda_{y}=-y^{\frac{1-\sigma}{2 \sigma}}\left(1+\lambda^{2}\right)^{\frac{3 \sigma-1}{2 \sigma}} \\
-y^{-1} \lambda\left(1+\lambda^{2}\right)\end{array}$ \\
\hline$w_{7}$ & $\left(e^{\varepsilon} x, e^{\varepsilon} u, e^{(1+\sigma) \varepsilon} t\right)$ & $\left(\dot{x}|t|^{\frac{-1}{\sigma+1}}, u|t|^{\frac{-1}{\sigma+1}}\right)$ & $\begin{aligned} v_{y y}= & \frac{\operatorname{sign}(t)}{(\sigma+1)^{\frac{1}{\sigma}}}\left(1+v_{y}^{2}\right)^{\frac{3 \sigma-1}{2 \sigma}} \\
& \cdot\left(v-y v_{y}\right)^{\frac{1}{\sigma}}\end{aligned}$ \\
\hline$w_{8}$ & $\left(\begin{array}{c}e^{\varepsilon}(x \cos \alpha \varepsilon-u \sin \alpha \varepsilon) \\
e^{\varepsilon}(u \cos \alpha \varepsilon+x \sin \alpha \varepsilon) \\
\left.e^{(\sigma+1) \varepsilon} t\right)\end{array}\right.$ & $\left|\begin{array}{c}\left(\left(u^{2}+x^{2}\right)|t|^{\frac{-2}{\sigma+1}}\right. \\
\left.\tan ^{-1} u / x-\frac{\alpha}{\sigma+1} \log |t|\right)\end{array}\right|$ & $\begin{array}{l}v_{y}=\frac{\lambda}{2 y} \\
2 \lambda_{y}=\frac{\operatorname{sign}(t)}{(\sigma+1)^{\frac{1}{\sigma}}} y^{\frac{1-\sigma}{2 \sigma}}(\alpha-\lambda)^{\frac{1}{\sigma}} \\
\left(1+\lambda^{2}\right)^{\frac{3 \sigma-1}{2 \sigma}}-y^{-1} \lambda\left(1+\lambda^{2}\right)\end{array}$ \\
\hline
\end{tabular}

\section{An optimal system for $\sigma=1 / 3$.}

Each element of $\mathfrak{s}(1 / 3)$ is a vector field in $(x, u, t) \in \mathbb{R}^{2}$. Since it is linear, it generates a global diffeomorphism on $\mathbb{R}^{2}$. The first five vectors, $\boldsymbol{v}_{1}$ to $\boldsymbol{v}_{5}$, are independent of $t$. They generate the special affine group in $\mathbb{R}^{2}$. On the other hand, $\boldsymbol{t}$ generates the diffeomorphism $T:(x, u, t) \rightarrow(x, u, t+\varepsilon), \varepsilon \in \mathbb{R}$, and $\boldsymbol{d}$ generates $D(x, u, t) \rightarrow\left(e^{\varepsilon} x, e^{\varepsilon} u, e^{4 / 3 \varepsilon} t\right)$. It is not hard to verify that any element in $S(1 / 3)$ can be written in the form $D T A$ for some $D, T$ and $A$, where $A$ is a special affine transformation.

We now introduce the vectors 


$$
\begin{aligned}
& \boldsymbol{w}_{9}=\boldsymbol{v}_{4} \\
& \boldsymbol{w}_{10}=\boldsymbol{v}_{3}-\boldsymbol{v}_{4}, \\
& \boldsymbol{w}_{11}=\boldsymbol{v}_{3}+\boldsymbol{v}_{4}+\boldsymbol{v}_{1}, \\
& \boldsymbol{w}_{12}=\boldsymbol{v}_{4}+\boldsymbol{t} \\
& \boldsymbol{w}_{13}=\boldsymbol{v}_{3}+\boldsymbol{v}_{4}+\boldsymbol{t}, \\
& \boldsymbol{w}_{14}=\boldsymbol{v}_{3}+\boldsymbol{v}_{4}-\boldsymbol{t}, \\
& \boldsymbol{w}_{15}=\boldsymbol{v}_{3}+\boldsymbol{v}_{4}+\boldsymbol{v}_{1}+\boldsymbol{t}, \\
& \boldsymbol{w}_{16}=\boldsymbol{v}_{3}+\boldsymbol{v}_{4}+\boldsymbol{v}_{1}-\boldsymbol{t}, \\
& \boldsymbol{w}_{17}=\boldsymbol{d}+\boldsymbol{v}_{4}, \\
& \boldsymbol{w}_{18}=\boldsymbol{d}+\boldsymbol{v}_{4}+\boldsymbol{v}_{2}, \\
& \boldsymbol{w}_{19}=\boldsymbol{d}+\alpha \boldsymbol{v}_{4}(\alpha>0, \alpha \neq 1), \text { and } \\
& \boldsymbol{w}_{20}=\boldsymbol{d}+\boldsymbol{v}_{3}+\boldsymbol{v}_{4} .
\end{aligned}
$$

Theorem 2.1. An optimal system of one-dimensional subalgebras consists of the family $\left\{\boldsymbol{w}_{i}, i=1, \ldots, 20\right\}$.

Proof. The action of the adjoint representation of $S(1 / 3)$ on $\mathfrak{s}(1 / 3)$ is given in Table 2. Let

$$
\boldsymbol{v}=\sum_{i=1}^{5} a_{i} \boldsymbol{v}_{i}+a_{6} \boldsymbol{t}+a_{7} \boldsymbol{d}
$$

be in $\mathfrak{s}(1 / 3)$. First of all, let us observe that by using $A d\left(\exp \varepsilon \boldsymbol{v}_{3}\right)$ we may rotate $\boldsymbol{v}_{4}$ and $\boldsymbol{v}_{5}$. As a result, we shall always assume that $a_{5}=0$ in the following discussion.

Case 1. $a_{3}^{2}>a_{4}^{2}$ or $a_{3}=a_{4}=0$.

We can use $A d\left(\exp \varepsilon \boldsymbol{v}_{5}\right)$ for a suitable $\varepsilon$ to eliminate $a_{4}$ while keeping $a_{5}=0$. Then $\langle\boldsymbol{v}\rangle$ is equivalent to a subspace spanned by $\left\{\boldsymbol{v}_{1}, \boldsymbol{v}_{2}, \boldsymbol{v}_{3}, \boldsymbol{t}, \boldsymbol{d}\right\}$, which, is simply the non-affine case. Using the same arguement as in the proof of Theorem 1.3 we conclude that $\langle\boldsymbol{v}\rangle$ is equivalent to the subalgebra generated by one of the vectors in $\left\{\boldsymbol{w}_{1}, \ldots, \boldsymbol{w}_{8}\right\}$. 
Case 2. $a_{3}^{2}=a_{4}^{2}>0$.

Using $A d\left(\exp \varepsilon \boldsymbol{v}_{3}\right)$, we may assume $a_{3}=a_{4}=1$. If $a_{4} \neq 0$, by $A d\left(\exp \varepsilon_{1} \boldsymbol{v}_{1}\right)$ and $A d\left(\exp \varepsilon_{2} \boldsymbol{v}_{2}\right)<\boldsymbol{v}>$ is equivalent to

$$
\left(a_{1}-\varepsilon_{1} a_{7}\right) \boldsymbol{v}_{1}+\left(a_{2}-\varepsilon_{2} a_{7}-2 \varepsilon_{1}\right) \boldsymbol{v}_{2}+\boldsymbol{v}_{3}+\boldsymbol{v}_{4}+a_{6} \boldsymbol{t}+a_{7} \boldsymbol{d},
$$

which, by choosing $\varepsilon_{1}=a_{1} / a_{7}$ and $\varepsilon_{2}=\left(a_{2}-2 a_{1} / a_{7}\right) / a_{7}$, becomes $\boldsymbol{v}_{3}+\boldsymbol{v}_{4}+a_{6} \boldsymbol{t}+a_{7} \boldsymbol{d}$. Using $A d(\exp \varepsilon \boldsymbol{t})$ to eliminate $\boldsymbol{t}$ and $A d\left(\exp \varepsilon \boldsymbol{v}_{5}\right)$ to normalize the resulting vector, we conclude that $\boldsymbol{v}$ is equivalent to

$$
d+v_{3}+v_{4}
$$

If $a_{7}=0$, use $A d\left(\exp \varepsilon \boldsymbol{v}_{1}\right)$ to eliminate $\boldsymbol{v}_{2}$ and then use $A d\left(\exp \varepsilon_{1} \boldsymbol{v}_{5}\right)$ and $A d\left(\exp \varepsilon_{2} d\right)$ to get

$$
e^{\varepsilon_{1}+\varepsilon_{2}} a_{1} \boldsymbol{v}_{1}+e^{-2 \varepsilon_{1}} \boldsymbol{v}_{3}+e^{-2 \varepsilon_{1}} \boldsymbol{v}_{4}+e^{\frac{4}{3} \varepsilon_{2}} a_{6} \boldsymbol{t}
$$

Since we can change $a_{1} \boldsymbol{v}_{1}+a_{3} \boldsymbol{v}_{3}+a_{4} \boldsymbol{v}_{4}+\boldsymbol{t} a_{6}$ into $-a_{1} \boldsymbol{v}_{1}+a_{3} \boldsymbol{v}_{3}+$ $a_{4} \boldsymbol{v}_{4}+a_{6} \boldsymbol{t}$ by $A d\left(\exp \pi v_{3}\right)$, we may assume without loss of generality that $a_{1} \geq 0$. If $a_{1}=0$, we see that $\boldsymbol{v}$ is equivalent to one of the following vectors:

$$
\begin{aligned}
& \boldsymbol{v}_{3}+\boldsymbol{v}_{4} \quad\left(\text { or } \boldsymbol{v}_{3}-\boldsymbol{v}_{4}\right) \\
& \boldsymbol{v}_{3}+\boldsymbol{v}_{4}+\boldsymbol{t}, \quad \text { or } \\
& \boldsymbol{v}_{3}+\boldsymbol{v}_{4}-\boldsymbol{t}
\end{aligned}
$$

if $a_{1}>0$ and $a_{6}=0, v$ is equivalent to

$$
v_{3}+v_{4}+v_{1}
$$

Finally, if $a_{1}>0$ and $a_{6} \neq 0$, we solve

$$
\begin{gathered}
e^{\varepsilon_{1}+\varepsilon_{2}}\left|a_{1}\right|=e^{-2 \varepsilon_{1}} \\
e^{\frac{4}{3} \varepsilon_{2}}\left|a_{6}\right|=e^{-2 \varepsilon_{1}}
\end{gathered}
$$

to determine $\varepsilon_{1}$ and $\varepsilon_{2}$. Then $a_{1}=a_{3}=a_{4}=\left|a_{6}\right|$. After a normalization, $\boldsymbol{v}$ is equivalent to

$$
\begin{aligned}
& \boldsymbol{v}_{3}+\boldsymbol{v}_{4}+\boldsymbol{v}_{1}+\boldsymbol{t}, \text { or } \\
& \boldsymbol{v}_{3}+\boldsymbol{v}_{4}+\boldsymbol{v}_{1}-\boldsymbol{t} .
\end{aligned}
$$


Case 3. $a_{3}^{2}<a_{4}^{2}$.

We may choose $\varepsilon$ in $A d\left(\exp \varepsilon \boldsymbol{v}_{5}\right)$ to eliminate $\boldsymbol{v}_{3}$ while keeping $a_{4} \neq 0$. If $a_{7}=0$, we use $A d\left(\exp \varepsilon_{1} \boldsymbol{v}_{1}\right) A d\left(\exp \varepsilon_{2} \boldsymbol{v}_{2}\right)$ for suitable $\varepsilon_{1}, \varepsilon_{2}$ to eliminate $\boldsymbol{v}_{1}$ and $\boldsymbol{v}_{2}$ and then use $A d(\exp \varepsilon \boldsymbol{d})$ to normalize the resulting vector. We get

$$
\begin{array}{ll}
\boldsymbol{v}_{4}, & \text { or } \\
\boldsymbol{v}_{4}+\boldsymbol{t}, & \text { or } \\
\boldsymbol{v}_{4}-\boldsymbol{t} . &
\end{array}
$$

Notice that the last two vectors are equivalent via $A d\left(\exp \frac{\pi}{2} \boldsymbol{v}_{3}\right)$.

If $a_{7} \neq 0$, using $A d\left(\exp \frac{\pi}{2} v_{3}\right)$ to change the sign of $a_{4}$. We may assume $a_{7}=1$ and $a_{4}>0$. Then by $A d(\exp \varepsilon d)$ we may eliminate $\boldsymbol{t}$. A further action by $A d\left(\exp \varepsilon_{1} \boldsymbol{v}_{1}\right)$ and $A d\left(\exp \varepsilon_{2} \boldsymbol{v}_{2}\right)$ reduces $\boldsymbol{v}$ to

$$
\left(a_{1}-\varepsilon_{1}-\varepsilon_{2} a_{4}\right) \boldsymbol{v}_{1}+\left(a_{2}-\varepsilon_{2}-\varepsilon_{1} a_{4}\right) \boldsymbol{v}_{2}+a_{4} \boldsymbol{v}_{4}+\boldsymbol{d}
$$

If $a_{4} \neq 1$, we may choose $\varepsilon_{1}$ and $\varepsilon_{2}$ so that $\boldsymbol{v}$ is equivalent to

$$
\boldsymbol{d}+\alpha \boldsymbol{v}_{4}, \alpha>0, \alpha \neq 1
$$

If $a_{4}=1$, take $\varepsilon_{1}=0$ and $\varepsilon_{2}=a_{1}$ to get $\boldsymbol{d}+\boldsymbol{v}_{4}+a_{2} \boldsymbol{v}_{2}$.

Using $A d(\exp \varepsilon \boldsymbol{d})$ to do a normalization, $\boldsymbol{v}$ is seen to be equivalent to

$$
\begin{array}{ll}
\boldsymbol{d}+\boldsymbol{v}_{4}, & \text { or } \\
\boldsymbol{d}+\boldsymbol{v}_{4}+\boldsymbol{v}_{2}, & \text { or } \\
\boldsymbol{d}+\boldsymbol{v}_{4}-\boldsymbol{v}_{2} . &
\end{array}
$$

Notice that the last two vectors are related by $A d\left(\exp \pi v_{3}\right)$.

Thus we have shown that any one-dimensional subspace of $\mathfrak{s}(1 / 3)$ is equivalent to one of the subspaces spanned by $\left\{\boldsymbol{w}_{1}, \ldots, \boldsymbol{w}_{20}\right\}$. Now we are going to show that these subspaces are mutually inequivalent. Following [22], we shall establish this result by looking for invariants of the adjoint representation. Recall that a function $i: \mathfrak{s}(1 / 3) \rightarrow \mathbb{R}$ is an invariant if $i(A d g) \boldsymbol{v})=i(\boldsymbol{v})$ for all $\boldsymbol{v}$ in $\mathfrak{s}(1 / 3)$ and $g \in S(1 / 3)$.

Fact 1. $a_{7}$ is an invariant.

This is obvious. 
Fact 2. $a \equiv a_{3}^{2}-a_{4}^{2}-a_{5}^{2}$ is an invariant.

First of all, under the adjoint action of $\boldsymbol{v}_{1}, \boldsymbol{v}_{2}, \boldsymbol{t}$ and $\boldsymbol{d}$, the coefficients of $\boldsymbol{v}_{3}, \boldsymbol{v}_{4}$, and $\boldsymbol{v}_{5}$ do not change. Next, $A d\left(\exp \varepsilon \boldsymbol{v}_{3}\right)$ does not change $a_{3}$ and $a_{4}^{2}+a_{5}^{2}$, and hence $a$. On the other hand, $\operatorname{Ad}\left(\exp \varepsilon \boldsymbol{v}_{4}\right)$ does not change $a_{4}$ and $a_{3}^{2}-a_{5}^{2}$. Similarly, $a$ is unchanged under $A d\left(\exp \varepsilon \boldsymbol{v}_{5}\right)$. We conclude that $a$ is an invariant.

Fact 3. Let

$$
x=\operatorname{det}\left(\begin{array}{ll}
a_{1} & -a_{5}-a_{7} \\
a_{2} & -a_{3}-a_{4}
\end{array}\right), y=\operatorname{det}\left(\begin{array}{ll}
a_{1} & a_{3}-a_{4} \\
a_{2} & a_{5}-a_{7}
\end{array}\right) .
$$

Then

$$
b \equiv \begin{cases}1, & a_{7}^{2}+a=0,(x, y) \neq(0,0) \\ 0, & \text { otherwise }\end{cases}
$$

is an invariant. As $a_{7}^{2}+a$ is an invariant, it suffices to check $(x, y) \neq$ $(0,0)$ is invariant under $a_{7}^{2}+a=0$.

Under the action of $\boldsymbol{v}_{1}, \boldsymbol{v}$ goes over to a new vector whose coefficient satisfies

$$
\left(\begin{array}{l}
\tilde{a}_{1} \\
\tilde{a}_{2}
\end{array}\right)=\left(\begin{array}{l}
a_{1} \\
a_{2}
\end{array}\right)+\varepsilon\left(\begin{array}{c}
-a_{5}-a_{7} \\
-a_{3}-a_{4}
\end{array}\right) .
$$

So $(\tilde{x}, \tilde{y})=(x, y)$ by a direct verification. Similarly we can show that $(\tilde{x}, \tilde{y})=(x, y)$ under $A d\left(\exp \varepsilon \boldsymbol{v}_{2}\right)$. Denote $A d\left(\exp \varepsilon \boldsymbol{v}_{3}\right) \boldsymbol{v}$ by $\sum \tilde{a}_{i} \boldsymbol{v}_{i}+$ $\tilde{a}_{6} \boldsymbol{t}+\tilde{a}_{7} \boldsymbol{d}$.

Using

$$
\left(\begin{array}{l}
x \\
y
\end{array}\right)=\left(\begin{array}{ll}
-a_{1} & a_{2} \\
-a_{2} & -a_{1}
\end{array}\right)\left(\begin{array}{l}
a_{3} \\
a_{4}
\end{array}\right)+\left(\begin{array}{cc}
-a_{4} & a_{5} \\
a_{5} & a_{4}
\end{array}\right)\left(\begin{array}{l}
a_{1} \\
a_{2}
\end{array}\right),
$$

we have

$$
\left(\begin{array}{l}
\tilde{x} \\
\tilde{y}
\end{array}\right)=A\left(\begin{array}{cc}
-a_{1} & a_{2} \\
-a_{2} & -a_{1}
\end{array}\right)\left(\begin{array}{l}
a_{3} \\
a_{4}
\end{array}\right)+A^{2}\left(\begin{array}{cc}
-a_{4} & a_{5} \\
a_{5} & a_{4}
\end{array}\right) A\left(\begin{array}{l}
a_{1} \\
a_{2}
\end{array}\right),
$$


where

$$
A=\left(\begin{array}{cc}
\cos \varepsilon & -\sin \varepsilon \\
\sin \varepsilon & \cos \varepsilon
\end{array}\right) .
$$

One can check that

$$
A\left(\begin{array}{cc}
-a_{4} & a_{5} \\
a_{5} & a_{4}
\end{array}\right) A=\left(\begin{array}{cc}
-a_{4} & a_{5} \\
a_{5} & a_{4}
\end{array}\right) .
$$

It follows that $(\tilde{x}, \tilde{y})=(x, y)^{t} A$, and so $\tilde{x}^{2}+\tilde{y}^{2}=x^{2}+y^{2}$.

Using

$$
x+y=-\frac{1}{2} \operatorname{det}\left(\begin{array}{cc}
a_{1}+a_{2} & -2\left(a_{4}+a_{7}\right) \\
a_{1}-a_{2} & 2\left(a_{3}-a_{5}\right)
\end{array}\right) \text {, }
$$

and

$$
x-y=-\frac{1}{2} \operatorname{det}\left(\begin{array}{cc}
a_{1}+a_{2} & -2\left(a_{3}+a_{5}\right) \\
a_{1}-a_{2} & 2\left(a_{4}-a_{7}\right)
\end{array}\right) .
$$

We see that the coefficient of $\tilde{\boldsymbol{v}}=A d\left(\exp \varepsilon \boldsymbol{v}_{4}\right) \boldsymbol{v}$ satisfy

$$
\begin{aligned}
& \tilde{a}_{1} \pm \tilde{a}_{2}=\left(a_{1} \pm a_{2}\right) e^{ \pm \varepsilon}, \\
& \tilde{a}_{3} \pm \tilde{a}_{4}=\left(a_{3} \pm a_{5}\right) e^{ \pm 2 \varepsilon} \\
& \tilde{a}_{4}=a_{4}
\end{aligned}
$$

and

$$
\tilde{a}_{7}=a_{7}
$$

Therefore, $\tilde{x}+\tilde{y}=e^{-\varepsilon}(x+y)$ and $\tilde{x}-\tilde{y}=e^{\varepsilon}(x-y)$.

Under $A d\left(\exp \varepsilon \boldsymbol{v}_{5}\right)$ we have

$$
\begin{aligned}
& \tilde{a}_{1}=a_{1} e^{\varepsilon} \\
& \tilde{a}_{2}=a_{2} e^{-\varepsilon}, \\
& \tilde{a}_{3} \pm \tilde{a}_{4}=\left(a_{3} \pm a_{4}\right) e^{\mp 2 \varepsilon} .
\end{aligned}
$$

As a result, $\tilde{x}=e^{-\varepsilon} x$ and $\tilde{y}=e^{\varepsilon} y$.

Finally, it is easy to see that $A d(\exp \varepsilon t)$ keeps $(x, y)$ unchanged and $A d(\exp \varepsilon d)$ sends $(x, y)$ to $e^{\varepsilon}(x, y)$. We have verified that $b$ is an invariant. 
Fact 4.

$$
c \equiv \begin{cases}\operatorname{sign} a_{6}, & a_{7}=0 \\ 0, & a_{7} \neq 0\end{cases}
$$

is an invariant.

It is easily checked that the sign of $a_{6}$ does not change when $a_{7}=0$.

Fact 5.

$$
d \equiv \begin{cases}\operatorname{sign} a_{3}, & a \geq 0 \\ 0, & \text { otherwise }\end{cases}
$$

is an invariant.

It suffices to check the sign of $a_{3}$ is preserved under the adjoint action. When $a \geq 0$, the adjoint actions of $\boldsymbol{v}_{1}, \boldsymbol{v}_{2}, \boldsymbol{v}_{3}, \boldsymbol{t}$ and $\boldsymbol{d}$ do not change $a_{3}$. On the other hand, under $A d\left(\exp \varepsilon \boldsymbol{v}_{4}\right)$ we have

$$
\tilde{a}_{3}=a_{3} \cosh 2 \varepsilon\left(1+\frac{a_{5}}{a_{3}} \tanh 2 \varepsilon\right)
$$

(assuming $a_{3}$ is nonzero). Since $a_{3}^{2} \geq a_{4}^{2}+a_{5}^{2} \geq a_{5}^{2}$ and $|\tanh 2 \varepsilon|<$ 1 , sign $\tilde{a}_{3}$ must be equal to sign $a_{3}$. Similarly, one can show that sign $a_{3}$ is unchanged under $A d\left(\exp \varepsilon \boldsymbol{v}_{5}\right)$. When $a_{3}$ vanishes, we must have $a_{4}=a_{5}=0$ under $a \geq 0$. So $a_{3}=0$ is preserved. We have shown that $d$ is an invariant.

Fact 6.

$$
e \equiv \begin{cases}1, & a_{3}=a_{4}=a_{5}=a_{7}=0,\left(a_{1}, a_{2}\right) \neq(0,0) \\ 0, & \text { otherwise }\end{cases}
$$

is an invariant.

Since the simultaneous vanishing of $a_{3}, a_{4}, a_{5}$ and $a_{7}$ is preserved under the adjoint action, we shall verify the invariance of $e$ under this vanishing condition. First of all, the adjoint actions of $\boldsymbol{v}_{1}, \boldsymbol{v}_{2}, \boldsymbol{v}_{5}, \boldsymbol{t}$ and $\boldsymbol{d}$ clearly do not change $e$. On the other hand, $A d\left(\exp \varepsilon \boldsymbol{v}_{3}\right)$ keeps $a_{1}^{2}+a_{2}^{2}$ constant. Finally, $A d\left(\exp \varepsilon \boldsymbol{v}_{4}\right)$ preserves $a_{1}^{2}-a_{2}^{2}$. If $a_{1}^{2}-a_{2}^{2}$ is non-zero, trivially $\left(\tilde{a}_{1}, \tilde{a}_{2}\right) \neq(0,0)$. If $a_{1}=a_{2}$, (resp. $\left.a_{1}=-a_{2}\right)$, $\tilde{a}_{1}=\tilde{a}_{2}=a_{1} e^{\varepsilon}$ (resp. $\tilde{a}_{1}=-\tilde{a}_{2}=a_{1} e^{-\varepsilon}$ ). Hence $e$ is also unchanged. We have shown that $e$ is an invariant. 
Table 5 .

\begin{tabular}{|c|c|c|c|c|c|c|}
\hline & $a_{7}$ & $a$ & $b$ & $c$ & $d$ & $e$ \\
\hline $\boldsymbol{w}_{1}$ & 0 & 0 & 0 & 0 & 0 & 0 \\
\hline $\boldsymbol{w}_{2}$ & 0 & 0 & 0 & 1 & 0 & 0 \\
\hline $\boldsymbol{w}_{3}$ & 0 & 0 & 0 & 1 & 0 & 1 \\
\hline $\boldsymbol{w}_{4}$ & 0 & 1 & 0 & 0 & 1 & 0 \\
\hline $\boldsymbol{w}_{5}$ & 0 & 1 & 0 & 1 & 1 & 0 \\
\hline $\boldsymbol{w}_{6}$ & 0 & 1 & 0 & -1 & 1 & 0 \\
\hline $\boldsymbol{w}_{7}$ & 1 & 0 & 0 & 0 & 0 & 0 \\
\hline $\boldsymbol{w}_{8}$ & 1 & $\alpha^{2}$ & 0 & 0 & $\operatorname{sign} \alpha$ & 0 \\
\hline $\boldsymbol{w}_{9}$ & 0 & -1 & 0 & 0 & 0 & 0 \\
\hline $\boldsymbol{w}_{10}$ & 0 & 0 & 0 & 0 & 1 & 0 \\
\hline $\boldsymbol{w}_{11}$ & 0 & 0 & 1 & 0 & 1 & 0 \\
\hline $\boldsymbol{w}_{12}$ & 0 & -1 & 0 & 1 & 0 & 0 \\
\hline $\boldsymbol{w}_{13}$ & 0 & 0 & 0 & 1 & 1 & 0 \\
\hline $\boldsymbol{w}_{14}$ & 0 & 0 & 0 & -1 & 1 & 0 \\
\hline $\boldsymbol{w}_{15}$ & 0 & 0 & 1 & 1 & 1 & 0 \\
\hline $\boldsymbol{w}_{16}$ & 0 & 0 & 1 & -1 & 1 & 0 \\
\hline $\boldsymbol{w}_{17}$ & 1 & -1 & 0 & 0 & 0 & 0 \\
\hline $\boldsymbol{w}_{18}$ & 1 & -1 & 1 & 0 & 0 & 0 \\
\hline $\boldsymbol{w}_{19}$ & 1 & $-\alpha$ & 0 & 0 & 0 & 0 \\
\hline $\boldsymbol{w}_{20}$ & 1 & 0 & 0 & 0 & 1 & 0 \\
\hline
\end{tabular}

Now we put everything in Table 5. It is clear from this table that all $\boldsymbol{w}_{i}^{\prime} s$ are mutually inequivalent, and hence they form an optimal system. The proof of Theorem 2.1 is completed.

A powerful method of finding optimal systems was developed in a series of papers started with Patera-Winternitz-Zassenhaus [24]. One may use this approach to determine optimal systems for (3). Here our new approach is elementary and is inspired by the treatment on the same problem for the 
heat equation in Olver [22] (see also Chou-Li-Qu [11]). While the invariants are used in [22] to reduce the number of non-equivalent vectors, we further introduce more invariants to achieve a complete classification. This approach has the advantage that through the evaluation of the invariants (see Tables 3 and 5) one can write out the explicit equivalence between the subalgebras.

We may group the twenty families of vectors in the optimal system into three families:

$$
\begin{aligned}
& \left\{\boldsymbol{w}_{1}, \boldsymbol{w}_{4}, \boldsymbol{w}_{9}, \boldsymbol{w}_{10}, \boldsymbol{w}_{11}\right\} \\
& \left\{\boldsymbol{w}_{2}, \boldsymbol{w}_{3}, \boldsymbol{w}_{5}, \boldsymbol{w}_{6}, \boldsymbol{w}_{12}, \boldsymbol{w}_{13}, \boldsymbol{w}_{14}, \boldsymbol{w}_{15}, \boldsymbol{w}_{16}\right\} \\
& \left\{\boldsymbol{w}_{7}, \boldsymbol{w}_{8}, \boldsymbol{w}_{17}, \boldsymbol{w}_{18}, \boldsymbol{w}_{19}, \boldsymbol{w}_{20}\right\}
\end{aligned}
$$

In the first family the vectors do not contain the time variable. Hence the group acts trivially on the $t$-component. In the second family, translation in $t$ is presented but dilatation is absent in each of its vectors. The corresponding invariant solutions are eternal solutions. The third family consists of similarity solutions; all vectors therein contain the dilatation but not the time-translation. The group actions and reduced equations for the invariant solutions corresponding to $\boldsymbol{w}_{9}, \ldots$, and $\boldsymbol{w}_{20}$ are listed below. They are further classified and studied in [20] in some details. All reduced equations are second order ODE's. As the curve may be closed or has large total curvature, the solution (or its derivatives) to these equations often blows up. However, the Euclidean invariance of the flow ensures that the form of the ODE does not change under any change of coordinates. As a result, we may remove the singularities in derivatives by rotating the coordinates. In order to obtain the largest invariant curve we need to match invariant local graphs in different coordinates together and this is one of the main tasks in [20]. As an illustation we shall work out the matching process for the invariant curves corresponding to $\boldsymbol{w}_{12}$ in the next section.

$\boldsymbol{w}_{9}=u \partial_{x}+x \partial_{u}$

Group action: $(x, u, t) \rightarrow(x \cosh \varepsilon+u \sinh \varepsilon, u \cosh \varepsilon+x \sinh \varepsilon, t)$

Invariant: $y=t, v=u^{2}-x^{2}$

Invariant solutions: $v=v(y)$ satisfies $v_{y}=2 v^{1 / 3}$. Hence the solutions are $u^{2}=x^{2}+(3 t+\text { constant })^{3 / 2}$, i.e., propagating hyperbolas.

$\boldsymbol{w}_{10}=-2 u \partial_{x}$

Group action: $(x, u, t) \rightarrow(x-2 \varepsilon u, u, t)$

Invariants: $y=t, v=u$.

Invariant solutioins: $v=v(y)$ satisfies $v_{y}=0$. Hence like $\boldsymbol{w}_{1}$, they are hor- 
izontal straight lines.

$\boldsymbol{w}_{11}=2 x \partial_{u}+\partial_{x}$

Group action: $(x, u, t) \rightarrow\left(x+\varepsilon, u+2 \varepsilon x+\varepsilon^{2}, t\right)$

Invariants: $y=t, v=u-x^{2}$.

Invariant solutions: $y=y(y)$ satisfies $v_{y}=2^{1 / 3}$. It leads to traveling waves $u=x^{2}+2^{1 / 3} t+$ constant, i.e., parabolas translating in constant speed.

$\boldsymbol{w}_{12}=x \partial_{u}+u \partial_{x}+\partial_{t}$

Group action: $(x, u, t) \rightarrow(x \cosh \varepsilon+u \sinh \varepsilon, x \sinh \varepsilon+u \cosh \varepsilon, t+\varepsilon)$

Invariants: $y=(u-x) e^{t}, v=(u+x) e^{-t}$

Invariant solutions: Let $u=e^{t} v-x$. Then $v=v(y)$ satisfies

$$
v_{y y}=\frac{1}{4}\left(v+y v_{y}\right)^{3}
$$

$\boldsymbol{w}_{13}, \boldsymbol{w}_{14}=2 x \partial_{u} \pm \partial_{t}$

Group action: $(x, u, t) \rightarrow(x, u+2 \varepsilon x, t \pm \varepsilon)$

Invariants: $y=x, v=u \mp 2 x t$

Invariant solutions : $u=v(y) \pm 2 x t$, where $v$ satisfies

$v_{y y}= \pm 8 y^{3}$

The invariant solutions for $\boldsymbol{w}_{13}, \boldsymbol{w}_{14}$ are explicitly given by

$$
u(x, t)= \pm \frac{2}{5} x^{5}+\left( \pm 2 t+C_{1}\right) x+C_{2}
$$

$\boldsymbol{w}_{15}, \boldsymbol{w}_{16}=2 x \partial_{u}+\partial_{x} \pm \partial_{t}$,

Group action: $(x, u, t) \rightarrow\left(x+\varepsilon, u+2 \varepsilon x+\varepsilon^{2}, t \pm \varepsilon\right)$

Invariants: $y=x \mp t, v=u-x^{2}$

Invariant solutions: $u=v(x-t)+x^{2}$, where $v$ satisfies $v_{y y}+2=\mp v_{y}^{3}$.

After this work was completed, Professor Olver kindly informed us that the invariant curves for $\boldsymbol{w}_{13}-\boldsymbol{w}_{14}$ were found in Calabi-Olver-Tannenbaum $[27]$.

$\boldsymbol{w}_{17}=\boldsymbol{d}+u \partial_{x}+x \partial_{u}$

Group action: $\quad(x, u, t) \rightarrow\left(e^{\varepsilon} \cosh \varepsilon x+e^{\varepsilon} \sinh \varepsilon u, e^{\varepsilon} \sinh \varepsilon x+\right.$ $\left.e^{\varepsilon} \cosh \varepsilon u, e^{\frac{4 \varepsilon}{3}} t\right)$

Invariants: $y=u-x, v=|t|^{-\frac{3}{2}}(u+x)$ 
Invariant solutions: $x=\frac{1}{2}\left(|t|^{\frac{2}{3}} v(y)-y\right), u=\frac{1}{2}\left(|t|^{\frac{2}{3}} v(y)+y\right)$, where $v$ satisfies

$$
\begin{aligned}
& v_{y y}=\frac{27}{32} v^{3} \quad(t>0), \text { or } \\
& v_{y y}=-\frac{27}{32} v^{3} \quad(t<0) .
\end{aligned}
$$

It is interesting to observe that in the contracting case $(t<0)$, any solutuion $v$ is periodic over the entire $y$-axis. So the invariant curve $\left(y, \frac{\sqrt{2}}{2}(-t)^{3 / 2} v\left(\frac{2 y}{\sqrt{2}}\right)\right.$ becomes zero at $t=0$. This solution was also described in Angenent-Sapiro-Tannenbaum [9].

$\boldsymbol{w}_{18}=\boldsymbol{d}+u \partial_{x}+x \partial_{u}+\partial_{u}$,

Group action: $(x, u, t) \rightarrow\left(\frac{1}{2}\left[e^{2 \varepsilon}\left(x+u+\frac{1}{2}\right)-\frac{1}{2}-(u-x-\varepsilon)\right]\right.$,

$$
\left.\frac{1}{2}\left[e^{2 \varepsilon}\left(x+u+\frac{1}{2}\right)-\frac{1}{2}+(u-x)+\varepsilon, e^{\frac{4 \varepsilon}{3}} t\right]\right) .
$$

Invariants: $y=u-x-\frac{3}{4} \log |t|, v=|t|^{-\frac{3}{2}}\left(u+x+\frac{1}{2}\right)$.

Invariant solutions: $u=|t|^{3 / 2} v(y)-x-1 / 2$ where $v$ satisfies

$$
\begin{aligned}
& v_{y y}=\frac{27}{256}(2 v-v y)^{3} \quad(t>0), \text { or } \\
& v_{y y}=-\frac{27}{256}(2 v-v y)^{3} \quad(t<0) .
\end{aligned}
$$

$\boldsymbol{w}_{19}=\boldsymbol{d}+\alpha\left(u \partial_{x}+x \partial_{u}\right)$

Group action: $(x, u, t) \rightarrow\left(e^{\varepsilon}(x \cosh \alpha \varepsilon+u \sinh \alpha \varepsilon)\right.$,

$$
\left.e^{\varepsilon}(u \cosh \alpha \varepsilon+x \sinh \varepsilon), e^{\frac{4 \varepsilon}{3}} t\right)
$$

Invariants: $y=|t|^{-\frac{3}{4}(1-\alpha)}(u-x), v=|t|^{-\frac{3}{4}(1+\alpha)}(u+x)$.

Invariant solutions: $x=\frac{1}{2}\left(|t|^{\frac{3}{4}(1+\alpha)} v(y)-|t|^{\frac{3}{4}(1-\alpha)} y\right)$,

$$
\begin{aligned}
& u=\frac{1}{2}\left(|t|^{\frac{3}{4}(1+\alpha)} v(y)+|t|^{\frac{3}{4}(1-\alpha)} y\right) \text { where } v \text { satisfies } \\
& v_{y y}=\frac{27}{256}\left[(1+\alpha) v-(1-\alpha) y v_{y}\right]^{3}(t>0), \text { or } \\
& v_{y y}=-\frac{27}{256}\left[(1+\alpha) v-(1-\alpha) y v_{y}\right]^{3}(t<0) .
\end{aligned}
$$


We point out that for some $\alpha \in(0,1)$, there corresponds a contracting $\boldsymbol{w}_{19}$-invariant solution which is a figure-eight [20].

$\boldsymbol{w}_{20}=\boldsymbol{d}+2 x \partial_{u}$

Group action: $(x, u, t) \rightarrow\left(x e^{\varepsilon},(u+2 \varepsilon x) e^{\varepsilon}, t e^{\frac{4}{3} \varepsilon}\right)$

Invariant: $y=x|t|^{-\frac{4}{3}}, v=\frac{u}{x}-\frac{3}{2} \log |t|$.

Invariant solutions: $u=\left(v\left(t^{-\frac{4}{3}} x\right)+\frac{3}{2} \log t\right)$ where $v$ satisfies

$$
\begin{aligned}
& y v_{y y}+2 v_{y}=\left[y\left(\frac{3}{2}-\frac{3}{4} y v_{y}\right)\right]^{3}(t>0), \text { or } \\
& y v_{y y}+2 v_{y}=-\left[y\left(\frac{3}{2}-\frac{3}{4} y v_{y}\right)\right]^{3}(t>0) .
\end{aligned}
$$

\section{Eternal solutions for $w_{12}$.}

We reduce (2.1) to a first order equation as follows. Let

$$
\left\{\begin{array}{l}
\xi=\log |y| \\
\eta=y v
\end{array}\right.
$$

Equation (2.1) goes over to

$$
\xi_{\eta \eta}=-3 \xi_{\eta}^{2}+2 \eta \xi_{\eta}^{3}-\frac{1}{4}
$$

The function $\omega=\xi_{\eta}$ satisifies

$$
\omega_{\eta}=-3 \omega^{2}+2 \eta \omega^{3}-\frac{1}{4}
$$

Now we classify the solutions of (3.3). First of all, the zero set of the right hand side of (3.3) can be expressed as the union of $\Lambda$ and $\Lambda^{\prime}$ where $\Lambda$ is the graph of a convex function $c=c(\eta)$ defined in $(0, \infty)$ satisfying

$$
\lim _{\eta \downarrow 0}\left(c(\eta)-\frac{3}{2 \eta}\right)=0
$$

and

$$
\lim _{\eta \rightarrow \infty}\left(c(\eta)-\frac{1}{2 \eta^{\frac{1}{3}}}\right)=0
$$


and, by the symmetry of $(3.3), \Lambda^{\prime}=\{(\eta,-c(-\eta)): \eta \in(-\infty, 0)\}$. Accordingly, any solution of (3.3) is increasing at points lying above $\Lambda$ or below $\Lambda^{\prime}$, and is decreasing in the region bounded between $\Lambda$ and $\Lambda^{\prime}$.

In the following lemmas, whose proofs are straightforward, $\omega(\eta)$ always is a solution of (3.3).

Lemma 3.1. For every positive $\eta_{0}$, there exists a sufficiently large $M_{0}$ such that, if $\left|\omega\left(\eta_{0}\right)\right| \geq M_{0}$, then $|\omega|$ blows up at some $\eta_{1}>\eta_{0}$. Furthermore, the blow-up point $\eta_{1}$ strictly decreases to $\eta_{0}$ as $\left|\omega\left(\eta_{0}\right)\right|$ strictly increases to infinity.

Lemma 3.2. If $\omega\left(\eta_{0}\right) \leq 1 / \eta_{0}$ (resp. $\left.\omega\left(\eta_{0}\right) \leq 1 / 2 \eta_{0}\right)$ for some $\eta_{0}>0$, then $\omega(\eta)<1 / \eta($ resp. $\omega(\eta)<1 / 2 \eta)$ for $\eta>\eta_{0}$.

Let $\omega$ be a solution of (3.3) satisfying $\omega(0)=\omega_{0} \geq 0$. By Lemma $3.1 \omega$ is a decreasing function defined in a maximal interval $(A, B),-\infty<A<$ $0<B<\infty$, satisfying

$$
\begin{aligned}
& \lim _{\eta \downarrow A} \omega(\eta)=\infty, \quad \text { and } \\
& \lim _{\eta \uparrow B} \omega(\eta)=-\infty
\end{aligned}
$$

By Lemma 3.2,

$$
\omega(\eta) \leq-2 \omega^{2}-\frac{1}{4} .
$$

Hence $\omega\left(\eta_{0}\right)=0$ for a unique $\eta_{0}>0$. By integrating (3.4) from 0 to $\eta_{0}$, we have

$$
\begin{aligned}
\eta_{0} & <\sqrt{2} \tan ^{-1} 2 \omega_{0} \\
& <\frac{\sqrt{2}}{2} \pi .
\end{aligned}
$$

Therefore, as $\omega_{0}$ increases from 0 to $\infty$, the zero points of the corresponding solutions strictly increase to a finite number $A^{*}$ not greater than $\sqrt{2} \pi / 2$. After passing the $\eta$-axis, $\omega$ becomes negative and blows up to $-\infty$ at some $\eta_{1}$. Using (3.4) one can see that these blow-up points have a finite supremum, say, $B^{*}$. We shall call any solution passing $\left(0, \omega_{0}\right), \omega_{0} \geq 0$, a type-I solution and the solution passing $\left(A^{*}, 0\right)$ and blows up at $\eta=B^{*}$ the typeA solution. It is clear that the type-A is the upper envelope of all type-I solutions. In particular, we know that it is defined over $\left(0, B^{*}\right)$ and blows up as $\eta$ tends to 0 . Call any solution passing $(A, 0), A>A^{*}$, a type-II solution. It is clear that the type-A solution is the separatrix between type-I 
and type-II solutions. Each type-II solution is decreasing over a maximal interval $(0, B), B>B^{*}$, and it satisfies

$$
\begin{aligned}
& \lim _{\eta \downarrow 0} \omega(\eta)=\infty, \quad \text { and } \\
& \lim _{\eta \uparrow B} \omega(\eta)=-\infty .
\end{aligned}
$$

All type-II solutions lie beneath $\Lambda$. Their supremum also defines a solution which we call the type-B solution. This curve is defined in $(0, \infty)$ and it satisfies

$$
\begin{aligned}
& \lim _{\eta \downarrow 0} \omega(\eta)=\infty, \quad \text { and } \\
& \lim _{\eta \rightarrow \infty} \omega(\eta)=0 .
\end{aligned}
$$

Finally, call any solution passing $\Lambda$ a type-III solution. It is defined over a maximal interval $(0, A), A>0$, decreasing all the way until it hits $\Lambda$, and then it becomes increasing and blows up at $A$. By Lemma 3.1 and Lemma 3.2 it is not hard to see that given any positive $A$, there exists a unique type-III solution which blows up at $A$. By the symmetry of the equation (3.3), $-\omega(-\eta)$ is a solution whenever $\omega(\eta)$ is a solution. We use this observation to define type- $\tilde{I},-\widetilde{I I},-\widetilde{I I I},-\tilde{A}$, and $-\tilde{B}$ solutions in an obvious way. It is easy to see that together with all type-I, -II, -III, -A and -B solutions, they form a foliation of the entire plane, provided the B-curve forms a separatrix between II- and III- curves. In fact, this is the content of the following lemma.

\section{Lemma 3.3.}

$$
\begin{aligned}
& \inf \{\omega(\eta): \omega \text { is a type-III solution }\} \\
& =\sup \{\omega(\eta): \omega \text { is a type-II solution }\}
\end{aligned}
$$

Proof. Denote the infimum and the supremum by $\omega_{1}$ and $\omega_{2}$ respectively. Both solutions satisfy

$$
\omega \rightarrow \frac{1}{2 \eta^{\frac{1}{3}}} \text { as } \eta \rightarrow \infty .
$$

If $\omega_{1}>\omega_{2}$, then

$$
\begin{aligned}
\frac{d}{d \eta} \log \left(\omega_{1}-\omega_{2}\right) & =-3\left(\omega_{1}+\omega_{2}\right)+2 \eta\left(\omega_{1}^{2}+\omega_{1} \omega_{2}+\omega_{2}^{2}\right) \\
& \geq \eta^{\frac{1}{3}}
\end{aligned}
$$


for large $\eta$. Therefore,

$$
\log \left(\omega_{1}-\omega_{2}\right)(\eta) \geq \frac{1}{2} \eta^{\frac{4}{3}}
$$

for large $\eta$, which is impossible. We conclude that $\omega_{1} \equiv \omega_{2}$.

For later use we must determine the asymptotic behavior of a solution at its end points. It is not hard to see that if $\omega(\eta)$ tends to infinity at some $\eta_{1} \neq 0$, then

$$
\lim _{\eta \rightarrow \eta_{1}} \omega(\eta)\left[4 \eta_{1}\left(\eta_{1}-\eta\right)\right]^{\frac{1}{2}}=1 .
$$

On the other hand, for the type-B solution,

$$
\lim _{\eta \rightarrow \infty} \omega(\eta) \eta^{\frac{1}{3}}=\frac{1}{2}
$$

It remains to study the asymptotic behavior of those solutions blowing up at $\eta=0$.

Lemma 3.4. If $\omega(\eta)$ tends to infinity as $\eta \downarrow 0$, then $\lim _{\eta \downarrow 0} \eta \omega(\eta)$ exists and is equal to 1 or $\frac{1}{2}$.

Proof. Denote $\zeta=\eta \omega$. We have

$$
\frac{d \zeta}{d \eta}=\frac{\zeta}{\eta}(2 \zeta-1)(\zeta-1)-\frac{1}{4} \eta
$$

The zero set of the right hand side of (3.7) consists of two disjoint parametrized curves given by

$$
\begin{aligned}
& \eta=2[\zeta(2 \zeta-1)(\zeta-1)]^{\frac{1}{2}}, \\
& \omega=\frac{\zeta}{2}[\zeta(2 \zeta-1)(\zeta-1)]^{-\frac{1}{2}},
\end{aligned}
$$

where $\zeta \in\left(0, \frac{1}{2}\right)$ and $\zeta>1$ respectively. Denote the former curve by $C_{1}$ and the latter by $C_{2}$. It is not hard to see that $C_{2}$ is the graph of a decreasing function over $(0, \infty)$, asymptotic to $\omega \eta=1$ as $\eta$ tends to zero. On the other hand, $C_{1}$ is the graph of a function over the $\omega$-axis, passing through the origin and is asymptotic to $\omega \eta=1 / 2$ as $\eta$ tends to zero. We observe that whenever a solution $\omega(\eta)$ passes $C_{1}$ or $C_{2}, d^{2} \zeta / d \eta^{2}=-1 / 2$. This means 
that for any solution $\omega, \eta \omega(\eta)$ must be eventually monotonic increasing or decreasing. Since near $\eta=0, \eta \omega(\eta)$ is bounded between 0 and $3 / 2$, the limit of $\eta \omega$ must exist as $\eta$ tends to zero. Letting $a=\lim _{\eta \downarrow 0} \eta \omega(\eta)$, it follows from (3.7) that $a(2 a-1)(a-1)=0$. If $a=0, \omega(\eta)$ must tend to infinity. However, then the function $h=1 / \omega$ satisfies

$$
h_{\eta}=3-2 \zeta+\frac{1}{4} h^{2} .
$$

So $h_{\eta}$ tends to 3 as $\eta \downarrow 0$. But then $\zeta$ tends to $1 / 3$, which leads to a contradiction. So $a$ must be equal to $\frac{1}{2}$ or 1 .

Using Lemma 3.2 and the fact that the type-A solution is the upper envelope of all type-I solutions, it follows from Lemma 3.4 that

$$
\lim _{\eta \downarrow 0} \eta \omega(\eta)=1 / 2
$$

for the type-A solution. It turns out that all other solutions behave in a different way.

Lemma 3.5. The solution satisfying (3.8) is unique.

Proof. Let $\omega_{1}$ and $\omega_{2}$ be two such solutions with $\omega_{2}>\omega_{1}$. Denote their inverses by $\eta_{1}$ and $\eta_{2}$ respectively. Then

$$
\begin{aligned}
\frac{d \log \left(\eta_{2}-\eta_{1}\right)}{d \omega} & =\frac{-2 \omega^{3}}{\left(-3 \omega^{2}+2 \eta_{2} \omega^{3}-\frac{1}{4}\right)\left(-3 \omega^{2}+2 \eta_{1} \omega^{3}-\frac{1}{4}\right)} \\
& \geq-3 / 4 \omega
\end{aligned}
$$

for large $\omega$. Therefore,

$$
\eta_{2}-\eta_{1} \geq c \omega^{-\frac{3}{4}}
$$

for some positive $c$. But, since $\eta_{1}-\eta_{2} \leq 1 / 2 \omega$, this is impossible.

Combining Lemmas 3.4 and 3.5, we know that any type-II, -B or -III solution must satisfy

$$
\lim _{\eta \downarrow 0} \eta \omega(\eta)=1
$$

Let us write

$$
\xi(\eta)=\eta(1-\eta \tau(\eta)) .
$$


Then $\tau$ satisfies the equation

$$
\tau_{\eta}=\frac{2 \tau^{2}}{1-\eta \tau}+\frac{1}{4}(1-\eta \tau)^{2} .
$$

This equation is uniquely solvable near $\eta=0$ subject to

$$
\tau(0)=\tau_{0} .
$$

We have

$$
\begin{aligned}
\omega(\eta) & =\frac{1}{\eta} \frac{1}{1-\eta \tau} \\
& =\frac{1}{\eta}\left(1+\tau_{0} \eta+o(\eta)\right) .
\end{aligned}
$$

As $\tau_{0}$ increases from $-\infty$ to $\infty, \omega$ runs through type-II, -B and -III solutions. Notice that the type-B solution corresponds to a positive $\tau_{0}^{*}$, since according to Lemma $3.2 \omega$ with non-positive $\tau_{0}$ must cross the positive $\eta$-axis.

Now all solutions $\xi$ to (3.2) can be obtained by integrating $\omega$. For each $\omega$ the solution $\xi$ is unique up to the addition of an arbitrary constant. In the following we fix a particular solution $\xi$ depending on the type of $\omega$.

For type-I solution $\omega$, we set

$$
\xi(\eta)=\int_{0}^{\eta} \omega(\tau) d \tau
$$

For the type-A solution,

$$
\xi(\eta)=\int_{1}^{\eta} \omega(\tau) d \tau
$$

For a type-II, -B or -III solution,

$$
\xi(\eta)=\log \eta+\int_{0}^{\eta}\left(\omega(\tau)-\frac{1}{\tau}\right) d \tau .
$$

Notice that by (3.10) $\xi$ is well-defined.

Let $\xi$ be any one of these solutions. Any $\boldsymbol{w}_{12}$-invariant curve is given in the form

$$
(v, y)=\left(\frac{\eta}{C e^{\xi(\eta)}}, C e^{\xi(\eta)}\right), \quad C \neq 0
$$


in the $(v, y)$-plane where $\eta$ is regarded as a parameter. Now we study the shapes of these solutions for different types of $\omega$. Clearly it suffices to concentrate on $C=1$. First, we have

$$
\begin{aligned}
& \frac{d v}{d \eta}=e^{-\xi}(1-\omega \eta), \quad \text { and } \\
& \frac{d y}{d \eta}=e^{\xi} \omega .
\end{aligned}
$$

Hence

$$
\frac{d y}{d v}=\frac{e^{2 \xi} \omega}{1-\omega \eta}
$$

and the curvature of the curve is give by

$$
k=\frac{-1}{4}\left[\eta^{2} \omega^{2}+\eta^{-2}(1-\omega \eta)^{2}\right]^{\frac{-3}{2}} .
$$

Combining our classification of the solutions of (3.3) with (3.11) and (3.12), we readily obtain the following result.

\section{Proposition 3.1.}

(i) Let $(v, y)$ be a type-I solution. Then $y=y(v)$ is a positive concave function over $[a, b]$, where $a=\eta_{0} e^{-\xi\left(\eta_{0}\right)}, b=\eta_{1} e^{-\xi\left(\eta_{1}\right)}$ and $\eta_{0}, \eta_{1}$ are the blow-up points of the corresponding $\omega$. It satisfies

(a) there is some $c \in(0, b)$ such that $y$ is strictly increasing in $[a, c)$ and is strictly decreasing in $(c, b]$;

(b) $d y / d v(a)>0$ and $d y / d v(b)<0$; and

(c) the curvature of $(v, y(v))$ vanishes only at $v=a, b$ and 0 .

(ii) Let $(v, y)$ be a type-A solution. Then $y=y(v)$ is a positive concave function in $(0, b]$ for $b=\eta_{1} e^{-\xi\left(\eta_{1}\right)}$, where $\eta_{1}$ is the blow-up point of the corresponding $\omega$. It satisfies

(a) there is some $c \in(0, b)$ such that $y$ is strictly increasing in $(0, c)$ and is strictly decreasing in $(c, b]$;

(b) $d y / d v(0)=1$ and $d y / d v(b)<0$; and

(c) the curvature of $(v, y(v))$ vanishes only at $v=b$ only. 
Proposition 3.2. Any type-II, $-B$ or -III solution is a concave arc emitting from $(1,0)$ and staying in $\{v>0, y>0\}$. Its other endpoint at $b=\eta_{1} e^{-\xi\left(\eta_{1}\right)}$, where $\eta_{1}$ is the blow-up point of the corresponding $\omega$, is the only inflecting point. Moreover,

(a) When the solution corresponds to a type-II solution with $\tau_{0} \leq 0$, the solution is a graph of $y=y(v)$ over $[1, b], b>1$, where $d y / d v(1)=$ $-1 / \tau_{0}$ and $d y / d v(b)<0$.

(b) When the solution corresponds to a type-II solution with $\tau_{0} \in\left(0, \tau_{0}^{*}\right)$, it can be decomposed into the graphs of $y_{1}(v)$ and $y_{2}(v)$, where $y_{1}$ is convex over some $[a, 1], a>0, d y_{1} / d v(a)=-\infty$ and $y_{2}$ is concave in $(a, b], d y_{2} / d v(b)<0$, and $y_{1}(a)=y_{2}(a)$.

(c) When the solution corresponds to a type-B solution, it is the graph of $y(v)$, a convex function over $(0,1]$ which satisfies $\lim _{v \downarrow 0} y(v)=\infty$ and $d y / d v(1)=-1 / \tau_{0}^{*}$.

(d) When the solution corresponds to a type-III solution, it is the graph of $y(v)$, a convex function over $[a, 1]$ for some $a>0 . y(v)$ is strictly decreasing in $[a, 1], d y / d v(a)<0$ and $d y / d v(1)=-1 / \tau_{0}$.

We have described the solution curves corresponding to all "normalized" $\xi^{\prime}$ s in these two propositions. Notice if $(v, y)$ is such a solution curve, $\left(\frac{\eta}{C y}, C y\right)$ is also a solution curve for any non-zero $C$, and it stays above or below the positive $v$-axis depending on whether $C$ is positive or negative. We call a solution curve of type- $\mathrm{I}^{ \pm}, \mathrm{A}^{ \pm}, \mathrm{II}^{ \pm}, \mathrm{B}^{ \pm}$or $\mathrm{III}^{ \pm}$in an obvious way. Similarly we define $\tilde{I}^{ \pm}-, \tilde{A}^{ \pm}-, \widetilde{I I}^{ \pm}-, \tilde{B}^{ \pm}$- and $\widetilde{I I I}{ }^{ \pm}$- solutions.

Starting with a piece of solution curve we shall show how to extend it to get a complete invariant curve. First, let us consider a $\mathrm{II}^{+}$-solution. We extend it from its right by a $\mathrm{III}^{+}$-solution. The right end point of this $\mathrm{III}^{+}-$ solution touches the $v$-axis. We extend it by a $\mathrm{II}^{-}$-solution, and then follows by a III $^{-}$-solution. The resulting curve hits the $v$-axis again, and we may extend it by a $\mathrm{II}^{+}$-solution. The procedure can be carried indefinitely to obtain a solution curve which is a graph over some interval $[A, B$ ). (In the following we shall show that $B=\infty$.) This semi-infinite invariant curve will be called a $\mathrm{II}^{+}$-tail. A similar construction will yield $\mathrm{II}^{-}-, \widetilde{I I}^{+}$- and $\widetilde{I I}^{-}$tails. 
Lemma 3.6. Let $y=y(v)$ be a solution of (3.11) which defines a tail in $[A, B)($ or $(B, A])$. Then $B=\infty($ or $-\infty)$ and $\lim _{v \rightarrow \infty} y(v)=0$ (or $\left.\lim _{v \rightarrow-\infty} y(v)=0\right)$.

Proof. Without loss of generality we assume the tail starts at $v=1$ with a $\mathrm{II}^{+}$-curve. Consider a piece of this tail which is a $\mathrm{II}^{+}$-curve followed by a $\mathrm{III}^{+}$-curve: $(v, y(v))$ is a $\mathrm{II}^{+}$-curve in $[a, c]$ and a $\mathrm{III}^{+}$-curve in $[c, d]$. We also let $b \in(a, c)$ be the maximum of $y$ in $[a, d]$. We claim

$$
\begin{aligned}
& |y(v)| \leq \sqrt[4]{8}\left|y_{v}(a)\right|^{\frac{1}{2}}, \quad \text { and } \\
& \left|y_{v}(v)\right| \leq\left|y_{v}(a)\right|
\end{aligned}
$$

for $v \in[a, d]$. For, from the equation

$$
y_{v v}=-\frac{1}{4}\left(y+v y_{v}\right)^{3},
$$

we have

$$
y_{v v}<-\frac{1}{4} y^{3}, \quad v \in(a, b) .
$$

Therefore,

$$
\frac{1}{2} y_{v}^{2}(v)+\frac{1}{16} y^{4}(v) \leq \frac{1}{2} y_{v}^{2}(a), \quad v \in(a, b) .
$$

On the other hand,

$$
y_{v v}>-\frac{1}{4} y^{3}, \quad v \in(b, c) .
$$

So

$$
\begin{aligned}
\frac{1}{2} y_{v}^{2}(v)+\frac{1}{16} y^{4}(v) & \leq \frac{1}{2} y_{v}^{2}(b)+\frac{1}{16} y^{4}(b) \\
& \leq \frac{1}{2} y_{v}^{2}(a)
\end{aligned}
$$

for $v \in[a, c]$. For $v \in(c, d), y$ is decreasing and convex. Therefore,

$$
|y(v)| \leq|y(c)|
$$

and

$$
\left|y_{v}(v)\right| \leq\left|y_{v}(c)\right|
$$

for $v \in(c, d)$. Combining (3.15), (3.16) and (3.17), we see that (3.13) holds. 
The inequalities in (3.13) imply that $y$ and $y_{v}$ are uniformly bounded for any tail in $[A, B)$. Since $y$ satisfies (3.14), we must have $B=\infty$. Let $a_{0}=1<a_{1}<a_{2}<\ldots$ be the left endpoints of the $\mathrm{II}^{+}$-curves forming the tail. We would like to show that $\left|y_{v}\left(a_{j}\right)\right| \rightarrow 0$ as $j \rightarrow \infty$. Together with (3.13) it implies the $C^{1}$-decay of the tail.

We observe that each $\mathrm{II}^{+}$-curve is headed by a $\mathrm{III}^{-}$-curve whose slope at $a_{j}$ is controlled by $1 / a_{j} \tau_{0}^{*}$. Therefore, as $a_{j} \rightarrow \infty$,

$$
\left|y_{v}\left(a_{j}\right)\right| \leq \frac{1}{a_{j} \tau_{0}^{*}} \rightarrow 0
$$

Now we can describe the invariant curves corresponding to $\boldsymbol{w}_{12}$. As we shall see, there are basically four types of them. Clearly it is sufficient to look at curves passing the point $(1,0)$. Let us consider a $\mathrm{II}^{+}$-tail starting at $(1,0)$ with slope $d y / d v(1)=-1 / \tau_{0}$. When the slope is equal to zero, the invariant curve is simply the $v$-axis. As the slope increases from 0 and remains small, we extend the $\mathrm{II}^{+}$-tail from $(1,0)$ by a type-III- ${ }^{-}$solution, and the type-III ${ }^{-}$solution is subsequently connected to a $\mathrm{II}^{-}$-tail and turns back. The resulting invariant complete curve is an immersed one, with two tails oscillating and converging to the $v$-axis as $v$ goes to infinity. When the slope increases further, the $\mathrm{II}^{+}$-tail will be connected to the type- $\mathrm{A}^{+}$ solution. By symmetry we obtain an invariant curve which is an odd function over the $v$-axis. After that the $\mathrm{II}^{+}$-tails will be connected to a type- $\mathrm{I}^{-}$ solution to form complete curves which are graphs of functions over $v$. It is worthwhile to see that there exists an even invariant curve which corresponds to $\omega$ with $\omega(0)=0$. When we increase the slope until $\tau_{0}=\tau_{0}^{*}$, the $\mathrm{II}^{+}-$ tail is connected to the type- $\mathrm{B}^{-}$solution at $(1,0)$. The resulting invariant curve is complete and is the graph of a function over $(0, \infty)$. When the slope increases further, the $\mathrm{II}^{+}$-tail is directly connected to a $\mathrm{II}^{-}$-tail. The resulting invariant curve has two tails, just like in the first case. When $\tau_{0}=0$, it is worthwhile to see that the invariant curve is symmetric with respect to the $v$-axis. When the slope goes beyond $\tau_{0}=0$, all invariant curves can be obtained by reflecting the four types of invariant curves with respect to the $v$-axis. Clearly, considering the extension from any other point on the $v$-axis yields essentially the same picture. Thus we have completely described the invariant curves for $\boldsymbol{w}_{12}$. See Figure 1 for some typical samples. 

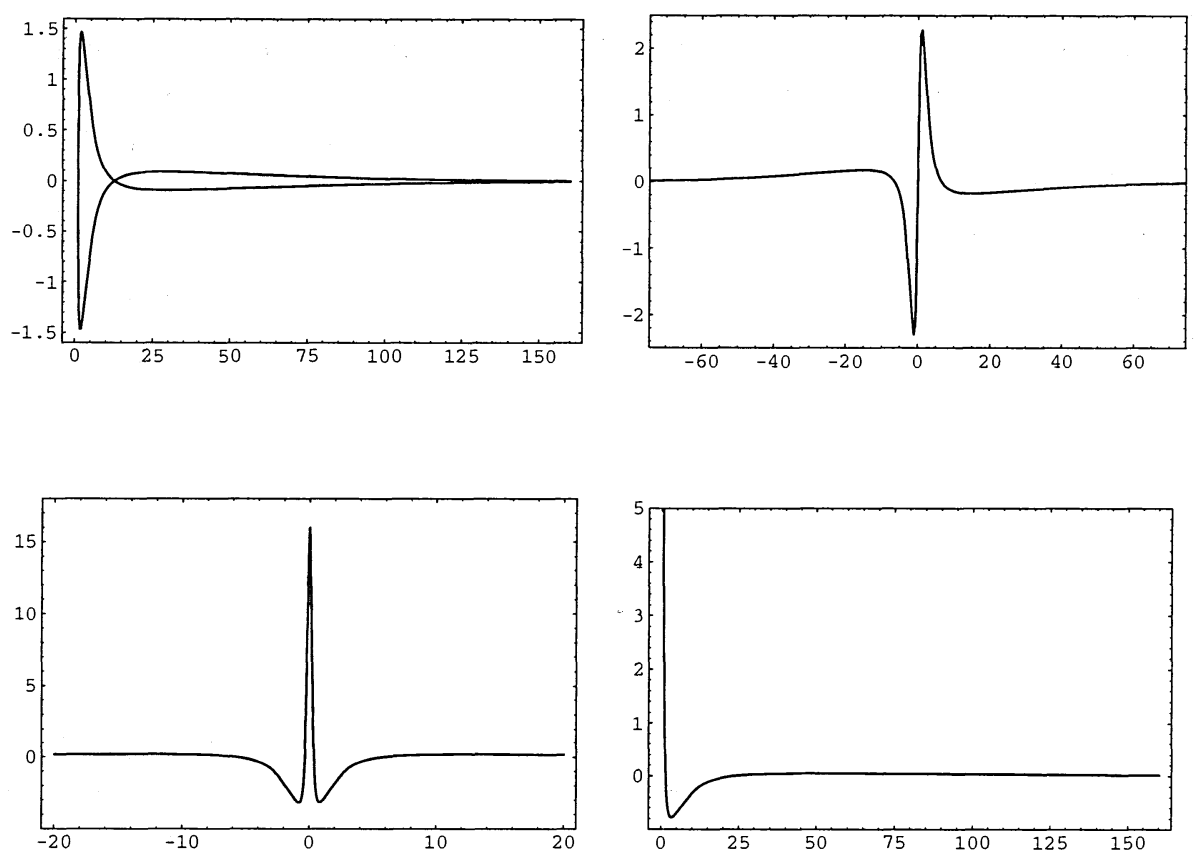

Figure 1: (a)-(d) Four types of eterenal $\boldsymbol{w}_{12}$-invariant curves.

\section{References.}

[1] U. Abresch and J. Langer, The normalized curve shortening flow and homothetic solutions, J. Differential Geom., 23 (1986), 175-196.

[2] S. Altschuler, Singularities of the curve shrinking flow for space curves, J. Differential Geom., 34 (1991), 491-514.

[3] L. Alvarez, F. Guichard, P.L. Lions and J.M. Morel, Axioms and fundamental equations of image processing, Arch. Rational Mech. Anal., 123 (1993), 199-257.

[4] B. Andrews, Evolving convex curves, Calc. Var. PDE's, 7 (1998), 315371.

[5] B. Andrews, Contracting of convex hypersurfaces by their affine normal, J. Differential Geom., 43 (1996), 207-230. 
[6] S. Angenent, Parabolic equations for curves on surfaces (I), Curves with p-integrable curvature, Ann. of Math., 132 (1990), 451-483.

[7] S. Angenent, Parabolic equations for curves on surfaces (II), Intersections, blow up and generalized solutions, Ann. of Math., 133 (1991), $171-215$.

[8] S. Angenent, On the formation of singularities in the curve shortening flow, J. Differential Geom., 33 (1991), 601-634.

[9] S.B. Angenent, G. Sapiro and A. Tannenbaum, On the affine heat equation for non-convex curves, J. Amer. Math. Soc., 11 (1998), 601-634.

[10] K.A. Brakke, The Motion of a Surface by its Mean Curvature, Princeton University Press, New Jercy, 1978.

[11] K.S. Chou, G. Li and C. Qu, Optimal systems for the heat equation, preprint 1999.

[12] K.S. Chou and X.P. Zhu, Shortening complete plane curves, J. Differential Geom., 50 (1998), 471-504.

[13] K.S. Chou and X.P. Zhu, The Curve Shortening Problem, Chapman \& Hall, CRC Press, London, 2000, in press.

[14] K. Ecker and G. Huisken, Mean curvature evolution of entire graphs, Ann. of Math., 130 (1989), 453-471.

[15] M. Gage and R. Hamilton, The heat equation shrinking convex plane curves, J. Differential Geom., 23 (1996), 69-96.

[16] M.A. Grayson, The heat equation shrinks embedded curves to round points, J. Differential Geom., 26 (1987), 285-314.

[17] R. Hamilton, Isoperimetric estimates for the curves shrinking flow in the plane, 201-222, in 'Modern Methods in Complex Analysis', The Princeton Conference in honor of Gunning and Kohn, ed. T. Bloom, et.al., Annals of Math, Studies, 137, Princeton Univ. Press, 1995.

[18] G. Huisken, Asymptotic behavior for singularities of the mean curvature flow, J. Differential Geom., 31 (1990), 285-299.

[19] N.H. Ibragimov, CRC Handbook of Lie Group Analysis of Differential Equations, 1, CRC Press, 1995. 
[20] G.X. Li, Group invariant solutions for some curvature driven flows, Ph.D. thesis, The Chinese University of Hong Kong, 1997.

[21] J.A. Oaks, Singularities and self intersections of curves evolving on surfaces, Indiana Univ. Math. J., 43 (1994), 959-981.

[22] P.J. Olver, Applications of Lie Groups to Differential Equation, second edition, Springer-Verlag, New York, 1993.

[23] L.V. Ovsiannikov, Group Analysis of Differential Equations, Academic Press, New York, 1982.

[24] J. Patera, P. Winternitz and H. Zassenhaus, Continuous subgroups of the fundamental groups of physics, I, General method and the Poincare groups, J. Math. Phys., 16 (1975), 1597-1614.

[25] G. Sapiro and T. Tannenbaum, On invariant curve evolution and image analysis, Indiana Univ. Math. J., 42 (1993), 985-1009.

[26] G. Sapiro and A. Tannenbaum, On affine plane curve evolution, J. Funct. Anal., 119 (1994), 79-120.

[27] E. Calabi, P.J. Olver and A. Tannenbaum, Affine geometry, curve flows and the invariant numerical approximations, Adv. Math., 124 (1996), 154-196.

Department of Mathematics

The Chinese University of Hong Kong

Shatin, Hong Kong

E-mail address: kschou@math.cuhk.edu.hk

E-mail address: gxli@math.cuhk.edu.hk

ReCEIVED OCTOBer 25, 2000. 